\title{
Amplitude analysis of hadron decays
}

\author{
R. Delbourgot and Dongsheng Liut \\ Physics Department, University of Tasmania, GPO Box 252C, Australia 7001
}

(May 17, 2018)

\begin{abstract}
We provide succinct covariant amplitude decompositions of 2-body weak hadronic decays, with which to compare data, including exclusive rates, helicity amplitudes and polarizations. For weak decays, the systematic dependence of these amplitudes on masses and quantum numbers of participating particles are determined within a factor of about two by the CKM angles and the Fermi constant so theoretical models need to be much more accurate if they are to be convincing.
\end{abstract}




\section{INTRODUCTION}

Weak nonleptonic processes constitute a sizeable fraction of the decay channels of recently discovered heavy charm and bottom mesons and baryons, not to mention strange hadrons. While a great deal is known about the semileptonic decays of those particles and much research [1] has been devoted to determining the hadronic matrix elements of the weak currents, we are rather less sure about the underlying picture [2] when no leptons emerge in the decay, because the strong interactions influence significantly the effective weak interactions: the gluons tend to muddy the current-current picture that governs semileptonic processes and it is not even certain that a factorization model [3] of four-quark operators prevails, especially for charmed states and heavy hadrons [4.

In order to gain some understanding of the dynamics governing nonleptonic decays it is helpful to express the decay amplitudes in some sort of standard kinematic form, with which to compare the theoretical predictions. This paper is devoted to expressing all 2-body nonleptonic amplitudes in the cleanest possible way, one which is neatly related to helicity amplitude decompositions. We will carry out our analysis in a covariant framework, since it is the easiest way to make contact with field theoretic descriptions in terms of various operators; an added advantage is that one can cross over to other channels easily, via simple substitution rules, and one need not worry about threshold factors since the latter come in naturally after contraction over external wavefunctions, provided one is careful not to introduce artificial kinematic singularities in the covariant decompositions. In principle one could extend the analysis to many-body non-resonant channels - abundant when the heavier hadrons decay - but really one requires some detailed knowledge of the 2-body hadron dynamics before one can make true progress on that wider front.

We shall consider the case of a particle/resonance of spin $j_{1}$, mass $m_{1}$ decaying into two lighter particles of spins and masses $j_{1}, j_{2}$ and $m_{2}, m_{3}$ respectively. The number of amplitudes $N$ required for describing the decay is given in the appendix and to each of them is associated some set of coupling constants $g_{i}^{j_{1} j_{2} j_{3}}\left(m_{1}, m_{2}, m_{3}\right)$. If one plots the $g$ in a three-dimensional graph as a function of its three mass arguments and because a physical process restricts us to the kinematic region $m_{1}>m_{2}+m_{3}$ (and its crossed versions, $m_{2}>m_{3}+m_{1}, m_{3}>m_{1}+m_{2}$ ), one will directly sample only a region of phase space - it lies outside the convex region bounded by the three planes joining pairs of lines $m_{1}=m_{2}, m_{2}=m_{3}, m_{3}=m_{1}$. We expect the couplings $g_{i}$ to depend quite substantially on the masses, because they are connected through the quantum numbers of the constituent quarks, if nothing else. 7 For large quantum number changes we anticipate big variations in the $g_{i}$ through the character of the CKM mixing matrix, but we expect the same thing to happen when higher radial or angular quantum numbers are excited as a consequence of the basic quark dynamics. There is quite of lot of data which lends iteslf to this kind of systematic analysis; however, if one wants to get inside the convex mass region, 2-body decays are useless: it becomes necessary to consider 3- or higher-body processes where the strong dynamics is known to be dominated by certain pole terms in various kinematical sections of the Dalitz plot and extrapolate to

\footnotetext{
${ }^{1}$ Sometimes the renormalization group can enlighten us about the variation with mass scale in a scheme like the standard model.
} 
those regions in order to pick out the residues and couplings. It is worth mentioning that the $g_{i}$ entering the effective Lagrangians cannot be real, because plain unitarity guarantees that their phase will be connected to the scattering amplitude of the outgoing hadrons at the very least. Nor does it follow that $g$ are necessarily real in the convex mass region, for it is sometimes possible that strong intermediate states (especially pions) arise in some decay channel below the exclusive mass threshold. In what follows we shall be careful not to assume that the $g$ are real.

We will focus attention on mesons with spins $j$ up to 1 and baryons with spins up to $3 / 2$, though it is quite easy to generalize the formulae below to situations when the initial particle has higher spin. Throughout we shall express the effective Lagrangian in the generic form

$$
\mathcal{L}^{j_{1} j_{2} j_{3}}=g^{j_{1} j_{2} j_{3}} \phi_{2}^{*}\left(p_{2}\right) \phi_{3}^{*}\left(p_{3}\right) \phi_{1}\left(-p_{1}\right)
$$

where we interpret $\phi$ as unity for spin 0 , as the polarization vector $\varepsilon^{\mu}$ for spin 1 , as the standard Dirac spinor $u$ for spin $1 / 2$ and as the Rarita-Schwinger spinor $u^{\mu}$ for spin $3 / 2$; for antifermions we replace incoming $u(p)$ by outgoing $v(p)$, in the standard way. It is also understood that there may arise several independent tensors, with associated coupling constants, which one may have to sum over. (See the Appendix for notational details.) To reiterate, the couplings clearly depend on the participating particle masses which are intimately tied to their quantum numbers, including internal ones like flavour. The aim of the exercise is to extract the couplings from the available data and look for systematic properties/deviations from symmetry predictions in order to pinpoint afterwards the underlying forces capable of producing such effective interactions.

The paper is divided into three parts. Section II deals with baryons: all their covariant decompositions and connections with helicity amplitudes. Section III contains the same tabulation for decaying mesons. Finally we look at some typical magnitudes in section IV, not only to exhibit the mass-dependence/quantum number relation, but to make the point that once phase-space and CKM angles are factored out, the couplings are all similar to within a factor of 2 ; this is is ultimately tied to the 4-quark current-current picture and it signifies that experimental models should aim for accuracy to better than $10 \%$ if they are to claim our attention. The purpose of this paper is to set the groundwork for a full investigation of experimentally measured exclusive decay processes [5] and thereby unravel the full form of the effective Lagrangian. This must then be converted into the form of multiquark operators, since this is the preferred way of probing the quark dynamics. The conversion requires specific knowledge of how the hadronic wavefunctions are built up from the quark field operators at that level and some sort of tracing formula. Be that as it

may, the emergent multiquark operators encapsulate the combined effect of weak and strong interactions.

\section{BARYON DECAY WIDTHS}

\section{A. Covariant Amplitudes}

We shall examine baryon decays where the outgoing baryon is identified with particle 2 and the meson with particle 3 ; in this paper we will focus on partial decay widths, rather than 
more refined observables like angular asymmetries and polarizations. We shall not assume anything about parity conservation since we are primarily interested in weak decays. The effective Lagrangian always takes the form, $\mathcal{L}=\phi^{*}\left(p_{3}\right) \bar{u}\left(p_{2}\right) \mathcal{M} u\left(-p_{1}\right)$ and $\mathcal{M}$ will contain two sets of opposite parity couplings which we label $f$ and $g$; the latter multiplies a negative parity quantity like $\gamma_{5}$ or the Levi-Civita symbol $\epsilon$. Remember that $-p_{1}$ is the physical incoming momentum, which is somewhat unconventional.

$1 / 2 \rightarrow 1 / 2+0$

The effective Lagrangian equals

$$
\mathcal{L}^{\frac{1}{2} \frac{1}{2} 0}=\bar{u}_{2}\left(f^{\frac{1}{2} \frac{1}{2} 0}+g^{\frac{1}{2} \frac{1}{2} 0} \gamma_{5}\right) u_{1} .
$$

The coupling constant $f^{\frac{1}{2} \frac{1}{2} 0}$ of the scalar interaction is associated with an $s$-wave amplitude, while $g^{\frac{1}{2} \frac{1}{2} 0}$ of the pseudoscalar interaction is tied to a $p$-wave one. In the resulting decay width,

$$
\begin{gathered}
\Gamma^{\frac{1}{2} \frac{1}{2} 0}=\frac{\Delta}{8 \pi m_{1}^{3}}\left[\left(m_{1} m_{2}-p_{1} \cdot p_{2}\right)\left|f^{\frac{1}{2} \frac{1}{2} 0}\right|^{2}-\left(m_{1} m_{2}+p_{1} \cdot p_{2}\right)\left|g^{\frac{1}{2} \frac{1}{2} 0}\right|^{2}\right] \\
\text { where } \quad \Delta^{2} \equiv\left[m_{1}^{2}-\left(m_{2}+m_{3}\right)^{2}\right]\left[m_{1}^{2}-\left(m_{2}-m_{3}\right)^{2}\right]
\end{gathered}
$$

note that one can obtain the contribution of $g^{\frac{1}{2} \frac{1}{2} 0}$ from that of $f^{\frac{1}{2} \frac{1}{2} 0}$ by substituting $-m_{1} m_{2}$ for $m_{1} m_{2}$, with no interference between them. This is a recurrent feature.

Among a plethora of such decays are typically, $\Lambda \rightarrow N \pi$ and $\Lambda_{c} \rightarrow \Sigma \pi$. $1 / 2 \rightarrow 1 / 2+1$

We write the interaction Lagrangian in the Sachs form,

$$
\mathcal{L}^{\frac{1}{2} \frac{1}{2} 1}=\varepsilon_{3}^{\nu *} \bar{u}_{2}\left[\frac{1}{2}\left(p_{2}-p_{1}\right)_{\nu}\left(f_{E}^{\frac{1}{2} \frac{1}{2} 1}+g_{E}^{\frac{1}{2} \frac{1}{2} 1} \gamma_{5}\right)+\epsilon_{\nu \alpha \beta \rho} p_{1}^{\alpha} p_{2}^{\beta} \gamma^{\rho}\left(f_{M}^{\frac{1}{2} \frac{1}{2} 1} \gamma_{5}+g_{M}^{\frac{1}{2} \frac{1}{2} 1}\right)\right] u_{1} .
$$

The corresponding decay width assumes a pleasing diagonal form?,

$\Gamma^{\frac{1}{2} \frac{1}{2} 1}=\frac{\Delta^{3}}{32 \pi m_{1}^{3}}\left[\left(m_{1} m_{2}-p_{1} \cdot p_{2}\right)\left(\frac{\left|f_{E}^{\frac{1}{2} \frac{1}{2} 1}\right|^{2}}{m_{3}^{2}}+2\left|f_{M}^{\frac{1}{2} \frac{1}{2} 1}\right|^{2}\right)-\left(m_{1} m_{2}+p_{1} \cdot p_{2}\right)\left(\frac{\left|g_{E}^{\frac{1}{2} \frac{1}{2} 1}\right|^{2}}{m_{3}^{2}}+2\left|g_{M}^{\frac{1}{2} \frac{1}{2} 1}\right|^{2}\right)\right]$

When particle 3 is a photon, and the baryon masses $m_{1}$ and $m_{2}$ are unequal as is necessary for the decay to occur, gauge invariance requires $f_{E}^{\frac{1}{2} \frac{1}{2} 1}=g_{E}^{\frac{1}{2} \frac{1}{2} 1} \rightarrow 0$. As a result the radiative width simplifies to

$$
\Gamma^{\frac{1}{2} \frac{1}{2} 1} \rightarrow \frac{\left(m_{1}^{2}-m_{2}^{2}\right)^{3}}{16 \pi m_{1}^{3}}\left[\left(m_{1}+m_{2}\right)^{2}\left|f_{M}^{\frac{1}{2} \frac{1}{2} 1}\right|^{2}+\left(m_{1}-m_{2}\right)^{2}\left|g_{M}^{\frac{1}{2} \frac{1}{2} 1}\right|^{2}\right]
$$

with $f_{M}^{\frac{1}{2} \frac{1}{2} 1}$ or $g_{M}^{\frac{1}{2} \frac{1}{2} 1}$ coupling disappearing, depending on the relative parity between the baryons.

\footnotetext{
${ }^{2}$ If one invokes the standard vector $\gamma_{\mu}$ and axial vector $\gamma_{\mu} \gamma_{5}$ couplings plus magnetic moment $\sigma_{\mu \nu} p_{3}^{\nu}$ counterpart terms, instead of the Sachs kinematic covariants, interference terms abound.
} 
Examples of such processes are $\Sigma^{+} \rightarrow p \gamma, \Lambda_{b} \rightarrow \psi \Lambda, \Lambda_{c} \rightarrow p \phi$.

$3 / 2 \rightarrow 1 / 2+0$

Moving on to excited baryon decays, this first case involves two couplings corresponding to $d$ and $p$ wave amplitudes. The effective interaction can be written uniquely as

$$
\mathcal{L}^{\frac{3}{2} \frac{1}{2} 0}=\frac{1}{2}\left(p_{3}-p_{2}\right)_{\lambda} \bar{u}_{2}\left(f^{\frac{3}{2} \frac{1}{2} 0}+g^{\frac{3}{2} \frac{1}{2} 0} \gamma_{5}\right) u_{1}^{\lambda},
$$

and it is straightforward to calculate the decay width,

$$
\Gamma^{\frac{3}{2} \frac{1}{2} 0}=\frac{\Delta^{3}}{96 \pi m_{1}^{5}}\left[\left(m_{1} m_{2}-p_{1} \cdot p_{2}\right)\left|f^{\frac{3}{2} \frac{1}{2} 0}\right|^{2}-\left(m_{1} m_{2}+p_{1} \cdot p_{2}\right)\left|g^{\frac{3}{2} \frac{1}{2} 0}\right|^{2}\right] .
$$

The decays $\Omega \rightarrow \Lambda K$ and $\Xi^{*} \rightarrow \Lambda \pi$ illustrate this case.

$1 / 2 \rightarrow 3 / 2+0$

This is basically the same as the previous case, since it corresponds to the interchange $p_{1} \leftrightarrow p_{2}$, apart from the initial spin averaging factor. (Note that the triangular Kallen function $\Delta$ is symmetric under mass exchange.)

An example is $N(1440) \rightarrow \Delta \pi$.

$3 / 2 \rightarrow 1 / 2+1$

When the final meson is vectorial we come across six independent amplitudes, for which we adopt the following Lagrangian ,

$$
\begin{aligned}
\mathcal{L}^{\frac{3}{2} \frac{1}{2} 1}=\varepsilon_{3}^{\nu *} \bar{u}_{2} & {\left[\left(p_{1} \cdot p_{3} g_{\lambda \nu}-p_{3 \lambda} p_{1 \nu}\right)\left(f_{T}^{\frac{3}{2} \frac{1}{2} 1}+g_{T}^{\frac{3}{2} \frac{1}{2} 1} \gamma_{5}\right)+p_{3 \lambda} p_{1 \nu}\left(f_{L}^{\frac{3}{2} \frac{1}{2} 1}+g_{L}^{\frac{3}{2} \frac{1}{2} 1} \gamma_{5}\right)\right.} \\
& \left.+\frac{1}{2}\left(p_{3}-p_{2}\right)_{\lambda} \epsilon_{\nu \alpha \beta \rho} p_{2}^{\alpha} p_{3}^{\beta} \gamma^{\rho}\left(f_{M}^{\frac{3}{2} \frac{1}{2} 1} \gamma_{5}+g_{M}^{\frac{3}{2} \frac{1}{2} 1}\right)\right] u_{1}^{\lambda} .
\end{aligned}
$$

The decay width is quasidiagonal:

$$
\begin{aligned}
& \Gamma^{\frac{3}{2} \frac{1}{2} 1}=\frac{\Delta\left(m_{1} m_{2}-p_{1} \cdot p_{2}\right)}{96 \pi m_{1}^{3}} {\left[2\left(\Delta^{2}+6 m_{1}^{2} m_{3}^{2}\right)\left|f_{T}^{\frac{3}{2} \frac{1}{2} 1}\right|^{2}+\frac{\Delta^{4}}{4 m_{1}^{2} m_{3}^{2}}\left|f_{L}^{\frac{3}{2} \frac{1}{2} 1}\right|^{2}+\frac{\Delta^{4}}{2 m_{1}^{2}}\left|f_{M}^{\frac{3}{2} \frac{1}{2} 1}\right|^{2}\right.} \\
&\left.+2 \Delta^{2} \operatorname{Re}\left(f_{T}^{\frac{3}{2} \frac{1}{2} 1} f_{L}^{\frac{3}{2} \frac{1}{2} 1 *}\right)-2 \frac{\Delta^{2}}{m_{1}} p_{1} \cdot p_{3} \operatorname{Re}\left(i f_{T}^{\frac{3}{2} \frac{1}{2} 1^{*}} f_{M}^{\frac{3}{2} \frac{1}{2} 1}\right)\right] \\
&+m_{1} \leftrightarrow-m_{1}, f_{i} \leftrightarrow g_{i} .
\end{aligned}
$$

This result simplifies when a final state photon is produced because gauge invariance leads to $f_{L}^{\frac{3}{2} \frac{1}{2} 1}=g_{L}^{\frac{3}{2} \frac{1}{2} 1} \rightarrow 0$. We end up with the expression,

$$
\begin{aligned}
\Gamma^{\frac{3}{2} \frac{1}{2} 1} \rightarrow \frac{\left(m_{1}^{2}-m_{2}^{2}\right)^{3}}{384 \pi m_{1}^{3}} & \left\{\left(m_{1}+m_{2}\right)^{2}\left[\left|f_{T}^{\frac{3}{2} \frac{1}{2} 1}\right|^{2}+\frac{\left(m_{1}^{2}-m_{2}^{2}\right)^{2}}{4 m_{1}^{2}}\left|f_{M}^{\frac{3}{2} \frac{1}{2} 1}\right|^{2}-\frac{m_{1}^{2}-m_{2}^{2}}{2 m_{1}} \operatorname{Re}\left(i f_{M}^{\frac{3}{2} \frac{1}{2} 1} f_{T}^{\frac{3}{2} \frac{1}{2} 1 *}\right)\right]\right. \\
& \left.+\left(m_{1}-m_{2}\right)^{2}\left[\left|g_{T}^{\frac{3}{2} \frac{1}{2} 1}\right|^{2}+\frac{\left(m_{1}^{2}-m_{2}^{2}\right)^{2}}{4 m_{1}^{2}}\left|g_{M}^{\frac{3}{2} \frac{1}{2} 1}\right|^{2}-\frac{m_{1}^{2}-m_{2}^{2}}{2 m_{1}} \operatorname{Re}\left(i g_{M}^{\frac{3}{2} \frac{1}{2} 1} g_{T}^{\frac{3}{2} \frac{1}{2} 1 *}\right)\right]\right\}
\end{aligned}
$$

Examples of such decays are $\Delta \rightarrow N \gamma, \Xi^{*} \rightarrow \Xi \gamma$.

$1 / 2 \rightarrow 3 / 2+1$

This is basically the same as the previous case with permutation of momenta $p_{1}$ and $p_{3}$, but examples of this process from particle physics are hard to find. One which has been seen is $\Lambda_{c} \rightarrow \Delta \bar{K}^{*}$.

$\underline{3 / 2 \rightarrow 3 / 2+0}$ 
The last two cases we consider are transitions from excited to excited baryons. When the final meson has spin zero, the Lagrangian takes the form,

$$
\mathcal{L}^{\frac{3}{2} \frac{3}{2} 0}=\bar{u}_{2}^{\mu}\left[\left(f_{T}^{\frac{3}{2} \frac{3}{2} 0}+g_{T}^{\frac{3}{2} \frac{3}{2} 0} \gamma_{5}\right)\left(p_{1} \cdot p_{2} g_{\mu \lambda}-p_{1 \mu} p_{2 \lambda}\right)+\left(f_{L}^{\frac{3}{2} \frac{3}{2} 0}+g_{L}^{\frac{3}{2} \frac{3}{2} 0} \gamma_{5}\right) p_{1 \mu} p_{2 \lambda}\right] u_{1}^{\lambda},
$$

and the width is determined to be

$$
\begin{aligned}
\Gamma^{\frac{3}{2} \frac{3}{2} 0}=\frac{\Delta}{144 \pi m_{1}^{3}}\left(m_{1} m_{2}-p_{1} \cdot p_{2}\right) & \left\{\left[9\left(p_{1} \cdot p_{2}\right)^{2}+\left(2 m_{1} m_{2}-p_{1} \cdot p_{2}\right)^{2}\right]\left|f_{T}^{\frac{3}{2} \frac{3}{2} 0}\right|^{2}+\right. \\
& \left.\frac{\Delta^{4}}{4 m_{1}^{2} m_{2}^{2}}\left|f_{L}^{\frac{3}{2} \frac{3}{2} 0}\right|^{2}+\frac{\Delta^{2}}{m_{1} m_{2}}\left(2 m_{1} m_{2}-p_{1} \cdot p_{2}\right) \operatorname{Re}\left(f_{T}^{\frac{3}{2} \frac{3}{2} 0} f_{L}^{\frac{3}{2} \frac{3}{2} 0 *}\right)\right\} \\
& +m_{1} m_{2} \leftrightarrow-m_{1} m_{2}, \quad f_{i} \leftrightarrow g_{i} .
\end{aligned}
$$

Examples of these processes are rare. One is $N(1520) \rightarrow \Delta \pi$. $3 / 2 \rightarrow 3 / 2+1$

This vector channel brings in ten independent amplitudes. We write the effective interaction Lagrangian as

$$
\begin{aligned}
\mathcal{L}^{\frac{3}{2} \frac{3}{2} 1}=\varepsilon_{3}^{\nu *} \bar{u}_{2}^{\mu} & \left\{\frac{1}{2}\left(p_{3}-p_{2}\right)_{\lambda}\left(f_{1 T}^{\frac{3}{2} \frac{3}{2} 1}+g_{1 T}^{\frac{3}{2} \frac{3}{2} 1} \gamma_{5}\right)\left(p_{2} \cdot p_{3} g_{\mu \nu}-p_{2 \nu} p_{3 \mu}\right)\right. \\
& +\frac{1}{2}\left(p_{1}-p_{3}\right)_{\mu}\left(f_{2 T}^{\frac{3}{2} \frac{3}{2} 1}+g_{2 T}^{\frac{3}{2} \frac{3}{2} 1} \gamma_{5}\right)\left(p_{1} \cdot p_{3} g_{\nu \lambda}-p_{1 \nu} p_{3 \lambda}\right) \\
& +\frac{1}{2}\left(p_{2}-p_{1}\right)_{\nu}\left(f_{3 T}^{\frac{3}{2} 2} 1+g_{3 T}^{\frac{3}{2} 2} \gamma_{5}\right)\left(p_{1} \cdot p_{2} g_{\mu \lambda}-p_{1 \mu} p_{2 \lambda}\right) \\
& +\frac{1}{8}\left(p_{3}-p_{2}\right)_{\lambda}\left(p_{1}-p_{3}\right)_{\mu}\left(p_{2}-p_{1}\right)_{\nu}\left(f_{L}^{\frac{3}{2} \frac{3}{2} 1}+g_{L}^{\frac{3}{2} \frac{3}{2} 1} \gamma_{5}\right) \\
& \left.+g_{\mu \lambda} \epsilon_{\nu \alpha \beta \rho} p_{1}^{\alpha} p_{2}^{\beta} \gamma^{\rho}\left(f_{M}^{\frac{3}{2} \frac{3}{2} 1} \gamma_{5}+g_{M}^{\frac{3}{2} \frac{3}{2} 1}\right)\right\} u_{1}^{\lambda} .
\end{aligned}
$$

It is straightforward, though tedious, to work out the decay width which here reads

$$
\begin{aligned}
\Gamma^{\frac{3}{2} \frac{3}{2} 1}=\frac{\Delta^{3}\left(m_{1} m_{2}-p_{1} \cdot p_{2}\right)}{144 \pi m_{1}^{3}}\{ & \frac{1}{2 m_{1}^{2}}\left(\Delta^{2}+6 m_{2}^{2} m_{3}^{2}\right)\left|f_{1 T}^{\frac{3}{2} \frac{3}{2} 1}\right|^{2}+\frac{1}{2 m_{2}^{2}}\left(\Delta^{2}+6 m_{1}^{2} m_{3}^{2}\right)\left|f_{2 T}^{\frac{3}{2} \frac{3}{2} 1}\right|^{2} \\
& +\frac{1}{4 m_{3}^{2}}\left[9\left(p_{1} \cdot p_{2}\right)^{2}+\left(2 m_{1} m_{2}-p_{1} \cdot p_{2}\right)^{2}\right]\left|f_{3 T}^{\frac{3}{2} \frac{3}{2} 1}\right|^{2} \\
& +\frac{\Delta^{4}}{16 m_{1}^{2} m_{2}^{2} m_{3}^{2}}\left|f_{L}^{\frac{3}{2} \frac{3}{2} 1}\right|^{2}+\frac{1}{2}\left(10+\frac{\Delta^{2}}{m_{1}^{2} m_{2}^{2}}\right)\left|f_{M}^{\frac{3}{2} \frac{3}{2} 1}\right|^{2}- \\
& m_{1} m_{2}\left(2 m_{1} m_{2}-p_{1} \cdot p_{2}\right) \operatorname{Re}\left[\left(\frac{f_{1 T}^{\frac{3}{2}} \frac{3}{2}}{m_{1}^{2}}+\frac{f_{2 T}^{\frac{3}{2} \frac{3}{2}}}{m_{2}^{2}}-\frac{\Delta^{2}}{4 m_{1}^{2} m_{2}^{2}} \frac{f_{L}^{\frac{3}{2} \frac{3}{2} 1}}{m_{3}^{2}}\right) f_{3 T}^{\frac{3}{2} \frac{3}{2} 1 *}\right] \\
& -\left(3-2 \frac{p_{1} \cdot p_{2}}{m_{1} m_{2}}\right) \operatorname{Re}\left[i f_{M}^{\frac{3}{2} \frac{3}{2} 1}\left(\frac{p_{2} \cdot p_{3}}{m_{1}} f_{1 T}^{\frac{3}{2} \frac{3}{2} 1}+\frac{p_{1} \cdot p_{3}}{m_{2}} f_{2 T}^{\frac{3}{2} \frac{3}{2} 1}\right)^{*}\right] \\
& -\frac{\Delta^{2}-4 m_{3}^{2}\left(p_{1} \cdot p_{2}+2 m_{1} m_{2}\right)}{4 m_{1} m_{2}} \operatorname{Re}\left(f_{1 T}^{\frac{3}{2} \frac{3}{2} 1} f_{2 T}^{\frac{3}{2} \frac{3}{2} 1 *}\right) \\
& \left.-\frac{\Delta^{2}}{2} \operatorname{Re}\left[\left(\frac{f_{1 T}^{\frac{3}{2}} \frac{3}{2} 1}{m_{1}^{2}}+\frac{f_{2 T}^{\frac{3}{2}} \frac{3}{2} 1}{m_{2}^{2}}\right) f_{L}^{\frac{3}{2} \frac{3}{2} 1 *}\right]\right\} \\
& +m_{1} m_{2} \leftrightarrow-m_{1} m_{2}, \quad f_{i}^{\leftrightarrow} \leftrightarrow g_{i} .
\end{aligned}
$$

In the massless vector limit, gauge invariance gives rise to conditions $f^{\frac{3}{2} \frac{3}{2} 1}=f_{L}^{\frac{3}{2} \frac{3}{2} 1}=g^{\frac{3}{2} \frac{3}{2} 1}=$ $g_{L}^{\frac{3}{2}} \frac{3}{2} 1 \rightarrow 0$, so the decay width reduces to 


$$
\begin{aligned}
\Gamma^{\frac{3}{2} \frac{3}{2} 1} \rightarrow \frac{\left(m_{1}^{2}-m_{2}^{2}\right)^{3}\left(m_{1}+m_{2}\right)^{2}}{576 \pi m_{1}^{3}} & \left\{\left(m_{1}^{2}-m_{2}^{2}\right)^{2}\left(\frac{\mid f_{1 T}^{\frac{3}{2}} \frac{3}{2} 1}{m_{1}^{2}}+\frac{\left.\left|f_{2 T}^{\frac{3}{2}}\right|^{2}\right|^{2}}{m_{2}^{2}}\right)\right. \\
& +\left(8+\frac{m_{1}^{2}}{m_{2}^{2}}+\frac{m_{2}^{2}}{m_{1}^{2}}\right)\left|f_{M}^{\frac{3}{2} \frac{3}{2} 1}\right|^{2}-\frac{\left(m_{1}^{2}-m_{2}^{2}\right)^{2}}{2 m_{1} m_{2}} \operatorname{Re}\left(f_{1 T}^{\frac{3}{2} \frac{3}{2} 1} f_{2 T}^{\frac{3}{2} \frac{3}{2} 1 *}\right) \\
& \left.-\left(m_{1}^{2}-m_{2}^{2}\right)\left(3+\frac{m_{1}}{m_{2}}+\frac{m_{2}}{m_{1}}\right) \operatorname{Re}\left[i f_{M}^{\frac{3}{2} \frac{3}{2} 1}\left(\frac{f_{1 T}^{\frac{3}{2} \frac{3}{2} 1}}{m_{1}}-\frac{f_{2 T}^{\frac{3}{2} \frac{3}{2} 1}}{m_{2}}\right){ }^{*}\right]\right\} \\
& +m_{1} m_{2} \leftrightarrow-m_{1} m_{2}, \quad f_{i} \leftrightarrow g_{i} .
\end{aligned}
$$

We have been unable to spot any examples of such decays from Particle Data Tables.

\section{B. Helicity amplitudes}

The appendix contains the definition of the reduced helicity amplitudes $M_{\lambda_{2}, \lambda_{3}}$. They can be calculated in terms of the covariantly defined coupling constants $f^{j_{1} j_{2} j_{3}}$ and $g^{j_{1} j_{2} j_{3}}$ above by proceeding to the centre of mass frame where,

$$
-p_{1}=\left(m_{1} ; 0,0,0\right), \quad p_{2}=\left(E_{2} ; q \sin \theta, 0, q \cos \theta\right), \quad p_{3}=\left(E_{3} ;-q \sin \theta, 0,-q \cos \theta\right),
$$

and

$$
2 m_{1} E_{2}=m_{1}^{2}+m_{2}^{2}-m_{3}^{2}, \quad 2 m_{1} E_{3}=m_{1}^{2}+m_{3}^{2}-m_{2}^{2}, \quad 2 m_{1} q=\Delta\left(m_{1}^{2}, m_{2}^{2}, m_{3}^{2}\right) .
$$

One then carries out the contraction over wavefunctions for particular helicities, as needed, and compares against the expected result, viz. $d_{\lambda_{1} \lambda_{2}-\lambda_{3}}^{j_{1}}(\theta) M_{\lambda_{2}, \lambda_{3}}$. For that purpose one requires the polarization vectors

$$
\begin{gathered}
\varepsilon^{( \pm 1)}\left(-p_{1}\right)=(0 ; \mp 1,-i, 0) / \sqrt{2}, \quad \varepsilon^{(0)}\left(-p_{1}\right)=(0 ; 0,0,1), \\
\varepsilon^{( \pm 1) *}\left(p_{2}\right)=\varepsilon^{(\mp 1) *}\left(p_{3}\right)=(0 ; \mp \cos \theta, i, \pm \sin \theta) / \sqrt{2}, \\
\varepsilon^{(0)}\left(p_{2}\right)=\left(q ; E_{2} \sin \theta, 0, E_{2} \cos \theta\right) / m_{2}, \quad \varepsilon^{(0)}\left(p_{3}\right)=\left(q ;-E_{3} \sin \theta, 0,-E_{3} \cos \theta\right) / m_{3} .
\end{gathered}
$$

(Obviously we disregard the last two zero helicity vectors when photons are involved.)

In addition we shall need the specific forms for the particle and antiparticle wavefunctions, $u$ and $v$ respectively, in the centre of mass frame, when considering the decays of mesons into two fermions. In our notation, the transposes $(\sim)$ read:

$$
\begin{gathered}
\tilde{u}^{(1 / 2)}\left(-p_{1}\right)=\sqrt{2 m_{1}}(1,0,0,0) ; \quad \tilde{u}^{(-1 / 2)}\left(-p_{1}\right)=\sqrt{2 m_{1}}(0,1,0,0) ; \\
\tilde{u}^{(1 / 2)}\left(p_{2}\right)=\left(\sqrt{E_{2}+m_{2}} \cos \frac{\theta}{2}, \sqrt{E_{2}+m_{2}} \sin \frac{\theta}{2}, \sqrt{E_{2}-m_{2}} \cos \frac{\theta}{2}, \sqrt{E_{2}-m_{2}} \sin \frac{\theta}{2}\right) ; \\
\tilde{u}^{(-1 / 2)}\left(p_{2}\right)=\left(-\sqrt{E_{2}+m_{2}} \sin \frac{\theta}{2}, \sqrt{E_{2}+m_{2}} \cos \frac{\theta}{2}, \sqrt{E_{2}-m_{2}} \sin \frac{\theta}{2},-\sqrt{E_{2}-m_{2}} \cos \frac{\theta}{2}\right) ;
\end{gathered}
$$




$$
\begin{gathered}
\tilde{v}^{(1 / 2)}\left(p_{3}\right)=\left(-\sqrt{E_{3}-m_{3}} \cos \frac{\theta}{2},-\sqrt{E_{3}-m_{3}} \sin \frac{\theta}{2}, \sqrt{E_{3}+m_{3}} \cos \frac{\theta}{2}, \sqrt{E_{3}+m_{3}} \sin \frac{\theta}{2}\right) ; \\
\tilde{v}^{(-1 / 2)}\left(p_{3}\right)=\left(\sqrt{E_{3}-m_{3}} \sin \frac{\theta}{2},-\sqrt{E_{3}-m_{3}} \cos \frac{\theta}{2}, \sqrt{E_{3}+m_{3}} \sin \frac{\theta}{2},-\sqrt{E_{3}+m_{3}} \cos \frac{\theta}{2}\right) .
\end{gathered}
$$

We now have all the ammunition needed to extract the helicity-coupling relations, which are written below in specific parity combinations for convenience.

$1 / 2 \rightarrow 1 / 2+0$

The symmetric combination of helicity amplitudes under $\lambda_{2}, \lambda_{3} \leftrightarrow-\lambda_{2},-\lambda_{3}$ (which is connected with particular parity) takes the form

$$
\frac{1}{2}\left(M_{1 / 2,0}+M_{-1 / 2,0}\right)=\sqrt{2\left(m_{1} m_{2}-p_{1} \cdot p_{2}\right)} f^{\frac{1}{2} \frac{1}{2} 0},
$$

while the antisymmetric combination (with opposite parity) reads

$$
\frac{1}{2}\left(M_{1 / 2,0}-M_{-1 / 2,0}\right)=-\sqrt{-2\left(m_{1} m_{2}+p_{1} \cdot p_{2}\right)} g^{\frac{1}{2} \frac{1}{2} 0} .
$$

Remember that $m_{1} m_{2}+p_{1} \cdot p_{2}$ is negative in our convention: all momenta outgoing. $\underline{1 / 2 \rightarrow 1 / 2+1}$

$$
\begin{aligned}
& \frac{1}{2}\left(M_{1 / 2,0}+M_{-1 / 2,0}\right)=\sqrt{2\left(m_{1} m_{2}-p_{1} \cdot p_{2}\right)} \frac{\Delta}{2 m_{3}} f_{E}^{\frac{1}{2} \frac{1}{2} 1}, \\
& \frac{1}{2}\left(M_{1 / 2,1}+M_{-1 / 2,-1}\right)=-\sqrt{\left(m_{1} m_{2}-p_{1} \cdot p_{2}\right)} \Delta f_{M}^{\frac{1}{2} \frac{1}{2} 1}, \\
& \frac{1}{2}\left(M_{1 / 2,0}-M_{-1 / 2,0}\right)=i \sqrt{-2\left(m_{1} m_{2}+p_{1} \cdot p_{2}\right)} \frac{\Delta}{2 m_{3}} g_{E}^{\frac{1}{2} \frac{1}{2} 1}, \\
& \frac{1}{2}\left(M_{1 / 2,1}-M_{-1 / 2,-1}\right)=i \sqrt{-\left(m_{1} m_{2}+p_{1} \cdot p_{2}\right)} \Delta g_{M}^{\frac{1}{2}} \frac{1}{2} 1
\end{aligned}
$$

$\underline{3 / 2 \rightarrow 1 / 2+0}$

$$
\begin{gathered}
\frac{1}{2}\left(M_{1 / 2,0}+M_{-1 / 2,0}\right)=\sqrt{\frac{1}{3}\left(m_{1} m_{2}-p_{1} \cdot p_{2}\right)} \frac{\Delta}{m_{1}} f^{\frac{3}{2} \frac{1}{2} 0}, \\
\frac{1}{2}\left(M_{1 / 2,0}-M_{-1 / 2,0}\right)=i \sqrt{-\frac{1}{3}\left(m_{1} m_{2}+p_{1} \cdot p_{2}\right)} \frac{\Delta}{m_{1}} g^{\frac{3}{2} \frac{1}{2} 0} .
\end{gathered}
$$

$\underline{1 / 2 \rightarrow 3 / 2+0}$

$$
\frac{1}{2}\left(M_{1 / 2,0}+M_{-1 / 2,0}\right)=-\sqrt{\frac{1}{3}\left(m_{1} m_{2}-p_{1} \cdot p_{2}\right)} \frac{\Delta}{m_{2}} f^{\frac{1}{2} \frac{3}{2} 0}
$$




$$
\frac{1}{2}\left(M_{1 / 2,0}-M_{-1 / 2,0}\right)=-i \sqrt{-\frac{1}{3}\left(m_{1} m_{2}+p_{1} \cdot p_{2}\right)} \frac{\Delta}{m_{2}} g^{\frac{1}{2} \frac{3}{2} 0} .
$$

$\underline{3 / 2 \rightarrow 1 / 2+1}$

The symmetric combinations of helicity amplitudes are

$$
\begin{aligned}
& \frac{1}{2}\left(M_{1 / 2,-1}+M_{-1 / 2,1}\right)=-\sqrt{2\left(m_{1} m_{2}-p_{1} \cdot p_{2}\right)} p_{1} \cdot p_{3} f_{T}^{\frac{3}{2} \frac{1}{2} 1}, \\
& \frac{1}{2}\left(M_{1 / 2,1}+M_{-1 / 2,-1}\right)=-\sqrt{\frac{2}{3}\left(m_{1} m_{2}-p_{1} \cdot p_{2}\right)}\left(p_{1} \cdot p_{3} f_{T}^{\frac{3}{2} \frac{1}{2} 1}+\frac{\Delta^{2}}{2 m_{1}} f_{M}^{\frac{3}{2} \frac{1}{2} 1}\right), \\
& \frac{1}{2}\left(M_{1 / 2,0}+M_{-1 / 2,0}\right)=-\sqrt{\frac{4}{3}\left(m_{1} m_{2}-p_{1} \cdot p_{2}\right)}\left(m_{1} m_{3} f_{T}^{\frac{3}{2} \frac{1}{2} 1}+\frac{\Delta^{2}}{4 m_{1} m_{3}} f_{L}^{\frac{3}{2} \frac{1}{2} 1}\right),
\end{aligned}
$$

while the asymmetric ones are

$$
\begin{aligned}
& \frac{1}{2}\left(M_{1 / 2,-1}-M_{-1 / 2,1}\right)=-i \sqrt{-2\left(m_{1} m_{2}+p_{1} \cdot p_{2}\right)} p_{1} \cdot p_{3} g_{T}^{\frac{3}{2} \frac{1}{2} 1}, \\
& \frac{1}{2}\left(M_{1 / 2,1}-M_{-1 / 2,-1}\right)=-i \sqrt{-\frac{2}{3}\left(m_{1} m_{2}+p_{1} \cdot p_{2}\right)}\left(p_{1} \cdot p_{3} g_{T}^{\frac{3}{2} \frac{1}{2} 1}+\frac{\Delta^{2}}{2 m_{1}} g_{M}^{\frac{3}{2} \frac{1}{2} 1}\right), \\
& \frac{1}{2}\left(M_{1 / 2,0}-M_{-1 / 2,0}\right)=-i \sqrt{-\frac{4}{3}\left(m_{1} m_{2}+p_{1} \cdot p_{2}\right)}\left(m_{1} m_{3} g_{T}^{\frac{3}{2} \frac{1}{2} 1}+\frac{\Delta^{2}}{4 m_{1} m_{3}} g_{L}^{\frac{3}{2} \frac{1}{2} 1}\right) .
\end{aligned}
$$

$\underline{1 / 2 \rightarrow 3 / 2+1}$

The various well-defined parity combinations are:

$$
\begin{aligned}
& \frac{1}{2}\left(M_{3 / 2,1}+M_{-3 / 2,-1}\right)=\sqrt{2\left(m_{1} m_{2}-p_{1} \cdot p_{2}\right)} p_{2} \cdot p_{3} f_{T}^{\frac{1}{2} \frac{3}{2} 1}, \\
& \frac{1}{2}\left(M_{1 / 2,1}+M_{-1 / 2,-1}\right)=\sqrt{\frac{2}{3}\left(m_{1} m_{2}-p_{1} \cdot p_{2}\right)}\left(p_{2} \cdot p_{3} f_{T}^{\frac{1}{2} \frac{3}{2} 1}-\frac{\Delta^{2}}{2 m_{2}} f_{M}^{\frac{1}{2} \frac{3}{2} 1}\right), \\
& \frac{1}{2}\left(M_{1 / 2,0}+M_{-1 / 2,0}\right)=\sqrt{\frac{4}{3}\left(m_{1} m_{2}-p_{1} \cdot p_{2}\right)}\left(m_{2} m_{3} f_{T}^{\frac{1}{2} \frac{3}{2} 1}+\frac{\Delta^{2}}{4 m_{2} m_{3}} f_{L}^{\frac{1}{2} \frac{3}{2} 1}\right), \\
& \frac{1}{2}\left(M_{3 / 2,1}-M_{-3 / 2,-1}\right)=i \sqrt{-2\left(m_{1} m_{2}+p_{1} \cdot p_{2}\right)} p_{2} \cdot p_{3} g_{T}^{\frac{1}{2} \frac{3}{2} 1}, \\
& \frac{1}{2}\left(M_{1 / 2,1}-M_{-1 / 2,-1}\right)=-i \sqrt{-\frac{2}{3}\left(m_{1} m_{2}+p_{1} \cdot p_{2}\right)}\left(p_{2} \cdot p_{3} g_{T}^{\frac{1}{2} \frac{3}{2} 1}+\frac{\Delta^{2}}{2 m_{2}} g_{M}^{\frac{1}{2} \frac{3}{1} 1}\right), \\
& \frac{1}{2}\left(M_{1 / 2,0}-M_{-1 / 2,0}\right)=i \sqrt{-\frac{4}{3}\left(m_{1} m_{2}+p_{1} \cdot p_{2}\right)}\left(m_{2} m_{3} g_{T}^{\frac{1}{2} \frac{3}{2} 1}+\frac{\Delta^{2}}{4 m_{2} m_{3}} g_{L}^{\frac{1}{2} \frac{3}{2} 1}\right) .
\end{aligned}
$$




$$
\begin{aligned}
& \underline{3 / 2 \rightarrow 3 / 2+0} \\
& \frac{1}{2}\left(M_{3 / 2,0}+M_{-3 / 2,0}\right)=-\sqrt{2\left(m_{1} m_{2}-p_{1} \cdot p_{2}\right)} p_{1} \cdot p_{2} f_{T}^{\frac{3}{2} \frac{3}{2} 0}, \\
& \frac{1}{2}\left(M_{1 / 2,0}+M_{-1 / 2,0}\right)=-\sqrt{\frac{2}{9}\left(m_{1} m_{2}-p_{1} \cdot p_{2}\right)}\left(\left(p_{1} \cdot p_{2}-2 m_{1} m_{2}\right) f_{T}^{\frac{3}{2} \frac{3}{2} 0}-\frac{\Delta^{2}}{2 m_{1} m_{2}} f_{L}^{\frac{3}{2} \frac{3}{2} 0}\right), \\
& \frac{1}{2}\left(M_{3 / 2,0}-M_{-3 / 2,0}\right)=-i \sqrt{-2\left(m_{1} m_{2}+p_{1} \cdot p_{2}\right)} p_{1} \cdot p_{2} g_{T}^{\frac{3}{2} \frac{3}{2} 0}, \\
& \frac{1}{2}\left(M_{1 / 2,0}-M_{-1 / 2,0}\right)=i \sqrt{-\frac{2}{9}\left(m_{1} m_{2}+p_{1} \cdot p_{2}\right)}\left(\left(p_{1} \cdot p_{2}+2 m_{1} m_{2}\right) g_{T}^{\frac{3}{2} \frac{3}{2} 0}+\frac{\Delta^{2}}{2 m_{1} m_{2}} g_{L}^{\frac{3}{2} \frac{3}{2} 0}\right) . \\
& \underline{3 / 2 \rightarrow 3 / 2+1} \\
& \frac{1}{2}\left(M_{3 / 2,1}+M_{-3 / 2,-1}\right)=\sqrt{\frac{1}{3}\left(m_{1} m_{2}-p_{1} \cdot p_{2}\right)} \Delta\left(\frac{p_{2} \cdot p_{3}}{m_{1}} f_{1 T}^{\frac{3}{2} \frac{3}{2} 1}+f_{M}^{\frac{3}{2}} \frac{3}{2} 1\right), \\
& \frac{1}{2}\left(M_{1 / 2,-1}+M_{-1 / 2,1}\right)=\sqrt{\frac{1}{3}\left(m_{1} m_{2}-p_{1} \cdot p_{2}\right)} \Delta\left(\frac{p_{1} \cdot p_{3}}{m_{2}} f_{2 T}^{\frac{3}{2} \frac{3}{2} 1}+f_{M}^{\frac{3}{2} \frac{3}{2} 1}\right), \\
& \frac{1}{2}\left(M_{1 / 2,1}+M_{-1 / 2,-1}\right)=\sqrt{\frac{1}{9}\left(m_{1} m_{2}-p_{1} \cdot p_{2}\right)} \Delta\left(\frac{p_{2} \cdot p_{3}}{m_{1}} f_{1 T}^{\frac{3}{2} \frac{3}{2} 1}+\frac{p_{1} \cdot p_{3}}{m_{2}} f_{2 T}^{\frac{3}{2}} \frac{3}{2} 1-2 \frac{p_{1} \cdot p_{2}}{m_{1} m_{2}} f_{M}^{\frac{3}{2} \frac{3}{2} 1}\right), \\
& \frac{1}{2}\left(M_{3 / 2,0}+M_{-3 / 2,0}\right)=-\sqrt{2\left(m_{1} m_{2}-p_{1} \cdot p_{2}\right)} \frac{\Delta}{2 m_{3}} p_{1} \cdot p_{2} f_{3 T}^{\frac{3}{2} \frac{3}{2} 1}, \\
& \frac{1}{2}\left(M_{1 / 2,0}+M_{-1 / 2,0}\right)=\sqrt{\frac{2}{9}\left(m_{1} m_{2}-p_{1} \cdot p_{2}\right)} m_{1} m_{2} m_{3} \Delta \\
& \left(\frac{f_{1 T}^{\frac{3}{2} \frac{3}{2} 1}}{m_{1}^{2}}+\frac{f_{2 T}^{\frac{3}{2} \frac{3}{2} 1}}{m_{2}^{2}}+\left(1-\frac{p_{1} \cdot p_{2}}{2 m_{1} m_{2}}\right) \frac{f_{3 T}^{\frac{3}{2} \frac{3}{2} 1}}{m_{3}^{2}}-\frac{\Delta^{2}}{4 m_{1}^{2} m_{2}^{2} m_{3}^{2}} f_{L}^{\frac{3}{2} \frac{3}{2} 1}\right)
\end{aligned}
$$

and 


$$
\begin{aligned}
& \frac{1}{2}\left(M_{3 / 2,1}-M_{-3 / 2,-1}\right)=i \sqrt{-\frac{1}{3}\left(m_{1} m_{2}+p_{1} \cdot p_{2}\right)} \Delta\left(\frac{p_{2} \cdot p_{3}}{m_{1}} g_{1 T}^{\frac{3}{2} \frac{3}{2} 1}+g_{M}^{\frac{3}{2} \frac{3}{2} 1}\right), \\
& \frac{1}{2}\left(M_{1 / 2,-1}-M_{-1 / 2,1}\right)=i \sqrt{-\frac{1}{3}\left(m_{1} m_{2}+p_{1} \cdot p_{2}\right)} \Delta\left(\frac{p_{1} \cdot p_{3}}{m_{2}} g_{2 T}^{\frac{3}{2} \frac{3}{2} 1}-g_{M}^{\frac{3}{2} \frac{3}{2} 1}\right), \\
& \frac{1}{2}\left(M_{1 / 2,1}-M_{-1 / 2,-1}\right)=i \sqrt{-\frac{1}{9}\left(m_{1} m_{2}+p_{1} \cdot p_{2}\right)} \Delta\left(-\frac{p_{2} \cdot p_{3}}{m_{1}} g_{1 T}^{\frac{3}{2} \frac{3}{2} 1}+\frac{p_{1} \cdot p_{3}}{m_{2}} g_{2 T}^{\frac{3}{2}} \frac{3}{2} 1-2 \frac{p_{1} \cdot p_{2}}{m_{1} m_{2}} g_{M}^{\frac{3}{2}} g^{\frac{3}{2} 1}\right) \\
& \frac{1}{2}\left(M_{3 / 2,0}-M_{-3 / 2,0}\right)=-i \sqrt{-2\left(m_{1} m_{2}+p_{1} \cdot p_{2}\right)} \frac{\Delta}{2 m_{3}} p_{1} \cdot p_{2} g_{3 T}^{\frac{3}{2} \frac{3}{2} 1}, \\
& \frac{1}{2}\left(M_{1 / 2,0}-M_{-1 / 2,0}\right)=i \sqrt{-\frac{2}{9}\left(m_{1} m_{2}+p_{1} \cdot p_{2}\right)} m_{1} m_{2} m_{3} \Delta \\
&\left(\frac{g_{1 T}^{\frac{3}{2}} \frac{3}{2} 1}{m_{1}^{2}}+\frac{g_{2 T}^{\frac{3}{2}} \frac{3}{2} 1}{m_{2}^{2}}+\left(1+\frac{p_{1} \cdot p_{2}}{2 m_{1} m_{2}}\right) \frac{g_{3 T}^{\frac{3}{2}} \frac{3}{2} 1}{m_{3}^{2}}-\frac{\Delta^{2}}{4 m_{1}^{2} m_{2}^{2} m_{3}^{2}} g_{L}^{\frac{3}{2} \frac{3}{2} 1}\right) .
\end{aligned}
$$

The exclusive decay rates for all of these processes can be found via the helicity amplitudes of course and, by comparison with the rates found in the covariant formalism, this provides an independent check on the correctness of the expressions connecting the two formalisms. It also allows us to deduce the density matrices, if needed, without recourse to further tedious evaluation of traces incorporating polarization projectors. It is worth remebering that to each helicity amplitude is associated a particular angular decay contribution.

\section{MESON DECAYS}

\section{A. Covariant amplitudes}

$\underline{0 \rightarrow 0+0}$

This is perfectly trivial. One only has

$$
\mathcal{L}^{000}=g^{000}
$$

leading to the decay width

$$
\Gamma^{000}=\frac{\Delta}{16 \pi m_{1}^{3}}\left|g^{000}\right|^{2} .
$$

Examples are the parity-conserving decay $\sigma \rightarrow \pi \pi$ and parity-violating decays like $K \rightarrow$ $\pi \pi$.

$\underline{0 \rightarrow 0+1}$

This too is effectively unique. We take

$$
\mathcal{L}^{001}=\frac{1}{2}\left(p_{2}-p_{1}\right)_{\nu} \varepsilon_{3}^{\nu *} g^{001}
$$


whereupon the resulting width equals

$$
\Gamma^{001}=\frac{\Delta^{3}}{64 \pi m_{1}^{3} m_{3}^{2}}\left|g^{001}\right|^{2}
$$

The processes $D \rightarrow \bar{K}^{*} \pi, \quad \phi \pi, \quad \bar{K}^{*} K$ exemplify this case.

$0 \rightarrow 1 / 2+1 / 2$

This can be obtained from the decay $1 / 2 \rightarrow 1 / 2+0$ by interchanging particles 1 and 3 , since the Lagrangian here reads,

$$
\mathcal{L}^{0 \frac{1}{2} \frac{1}{2}}=\bar{u}_{2}\left[f^{0 \frac{1}{2} \frac{1}{2}}+g^{0 \frac{1}{2} \frac{1}{2}} \gamma_{5}\right] v_{3}
$$

and by recalling the connection between $v$ and $u$, spelled out in the appendix. This implies that the sum over spins is obtainable by interchanging the labels 1 and 3, remembering also to multiply the conjugation factor $(-1)^{2 j}$. Upon including the correct phase space factor, this gives,

$$
\Gamma^{0 \frac{1}{2} \frac{1}{2}}=\frac{\Delta}{4 \pi m_{1}^{3}}\left[\left(p_{2} \cdot p_{3}-m_{2} m_{3}\right)\left|f^{0 \frac{1}{2} \frac{1}{2}}\right|^{2}+\left(p_{2} \cdot p_{3}+m_{2} m_{3}\right)\left|g^{0 \frac{1}{2} \frac{1}{2}}\right|^{2}\right] .
$$

One may safely apply this substitution rule to save repetitious calculations of spin sums in other instances. Hereafter we shall do this and suppress a host of similar results which can be read off from others.

Hadronic examples of these decays are very unusual. A rarity is $\eta_{c} \rightarrow N \bar{N}$. $\underline{0 \rightarrow 1+1}$

This time we encounter three independent amplitudes if we make no assumptions about parity. It corresponds to the number of ways that two spin 1 systems can be added. We write the effective Lagrangian in the form,

$$
\mathcal{L}^{011}=\varepsilon_{2}^{\mu *} \varepsilon_{3}^{\nu *}\left[g_{T}^{001}\left(g_{\mu \nu} p_{2} \cdot p_{3}-p_{2 \nu} p_{3 \mu}\right)+g_{L}^{011} p_{2 \nu} p_{3 \mu}+g_{M}^{011} \epsilon_{\mu \nu \alpha \beta} p_{2}^{\alpha} p_{3}^{\beta}\right]
$$

Summing over final spins, it is straightforward to work out the decay width,

$$
\Gamma^{011}=\frac{\Delta}{32 \pi m_{1}^{3}}\left[\left(\Delta^{2}+6 m_{2}^{2} m_{3}^{2}\right)\left|g_{T}^{011}\right|^{2}+\Delta^{2}\left|g_{M}^{011}\right|^{2}+\frac{\Delta^{4}}{8 m_{2}^{2} m_{3}^{2}}\left|g_{L}^{011}\right|^{2}+\Delta^{2} \operatorname{Re}\left(g_{T}^{011} g_{L}^{011 *}\right)\right] .
$$

If the decay is radiative, one of the final particles is a photon (say 3) and then gauge invariance requires $g_{L}^{011} \rightarrow 0$; the width reduces to

$$
\Gamma^{011} \rightarrow \frac{\Delta^{3}}{32 \pi m_{1}^{3}}\left(\left|g_{T}^{011}\right|^{2}+\left|g_{M}^{011}\right|^{2}\right), \quad \Delta \rightarrow m_{1}^{2}-m_{2}^{2}
$$

(Actually, one or other of the two couplings $g_{M}, g_{T}$ must be set to zero, since electromagnetism conserves parity.) It is even possible for both final particles to be photons, as in $\pi^{0}$ decay, in which case $\Delta$ simplifies further to $m_{1}^{2}$.

The decays $K \rightarrow \gamma \gamma, \quad \eta_{c} \rightarrow \rho \rho, \quad D \rightarrow K^{*} \rho$ typify this case.

$0 \rightarrow 1 / 2+3 / 2$

This case is obtainable $3 / 2 \rightarrow 1 / 2+0$ by substitution; remember to adjust the external spin average by a factor of 4 for the partial decay width. 
No examples of this process stand out in the Particle Data tables.

$0 \rightarrow 3 / 2+3 / 2$

Again we obtain this from $3 / 2 \rightarrow 3 / 2+0$ by substitution and insert an extra factor of 4 to make up for the spin average.

It will be a long time before such exclusive modes are detected. $1 \rightarrow 0+0$

The effective Lagrangian here is just a labelling permutation $(1 \leftrightarrow 3)$ of the previous process $0 \rightarrow 0+1$; thus we get

$$
\Gamma^{100}=\frac{\Delta^{3}}{192 \pi m_{1}^{5}}\left|g^{100}\right|^{2}
$$

where an extra factor $1 / 3$ accounts for the average over the spin states of the initial vector meson. The decays $\rho \rightarrow \pi \pi, \omega \rightarrow \pi \pi$ are typical.

$\underline{1 \rightarrow 0+1}$

The effective Lagrangian is obtainable from the earlier process, $0 \rightarrow 1+1$, by permuting the labels 1 and 2. Hence the decay is found to be

$$
\Gamma^{101}=\frac{\Delta}{96 \pi m_{1}^{3}}\left[\left(\Delta^{2}+6 m_{1}^{2} m_{3}^{2}\right)\left|g_{T}^{101}\right|^{2}+\Delta^{2}\left|g_{M}^{101}\right|^{2}+\frac{\Delta^{4}}{8 m_{1}^{2} m_{3}^{2}}\left|g_{L}^{101}\right|^{2}+\Delta^{2} \operatorname{Re}\left(g_{T}^{101} g_{L}^{101 *}\right)\right] .
$$

In particular, a radiative vector meson decay into a scalar meson requires $g_{L}^{101} \rightarrow 0$ in which case the width reduces to

$$
\Gamma^{101} \rightarrow \frac{\Delta^{3}}{96 \pi m_{1}^{3}}\left(\left|g_{T}^{101}\right|^{2}+\left|g_{M}^{101}\right|^{2}\right)
$$

with one of $g_{M}$ or $g_{T}$ vanishing by parity conservation. Examples of such vector decays are $\psi \rightarrow \omega \pi, \quad D^{*} \rightarrow D \gamma$.

Here we can point to $\phi \rightarrow \rho \pi, \quad B^{*} \rightarrow B \gamma$ as typical cases.

$1 \rightarrow 1 / 2+1 / 2$

This situation lends itself to the substitution rule, and apart from the spin averaging factor (namely a factor of $2 / 3$ ), can be derived directly from $1 / 2 \rightarrow 1 / 2+1$. It is

$\Gamma^{1 \frac{1}{2} \frac{1}{2}}=\frac{\Delta^{3}}{48 \pi m_{1}^{3}}\left[\left(m_{2} m_{3}-p_{2} \cdot p_{3}\right)\left(\frac{\left|f_{E}^{\frac{1}{2} \frac{1}{2} 1}\right|^{2}}{m_{1}^{2}}+2\left|f_{M}^{\frac{1}{2} \frac{1}{2} 1}\right|^{2}\right)-\left(m_{2} m_{3}+p_{2} \cdot p_{3}\right)\left(\frac{\left|g_{E}^{\frac{1}{2} \frac{1}{2} 1}\right|^{2}}{m_{1}^{2}}+2\left|g_{M}^{\frac{1}{2} \frac{1}{2} 1}\right|^{2}\right)\right]$

Typical examples are $\psi \rightarrow N \bar{N}, \Sigma \bar{\Sigma}$.

$\underline{1 \rightarrow 1+1}$

This is the most complicated purely mesonic process which we consider. It brings in seven independent amplitudes in general, no assumption being made about parity. The most elegant decomposition of the effective Lagrangian is

$$
\mathcal{L}^{111}=\varepsilon_{2}^{\mu *} \varepsilon_{3}^{\nu *} \mathcal{M}_{\lambda \mu \nu} \varepsilon_{1}^{\lambda}
$$

with 


$$
\begin{aligned}
& \mathcal{M}_{\lambda \mu \nu}=\frac{1}{2}\left(p_{3}-p_{2}\right)_{\lambda}\left[g_{1 T}^{111}\left(p_{2} \cdot p_{3} g_{\mu \nu}-p_{3 \mu} p_{2 \nu}\right)+g_{1 M}^{111} \epsilon_{\mu \nu \alpha \beta} p_{2}^{\alpha} p_{3}^{\beta}\right] \quad+ \\
& \frac{1}{2}\left(p_{1}-p_{3}\right)_{\mu}\left[g_{2 T}^{111}\left(p_{3} \cdot p_{1} g_{\nu \lambda}-p_{1 \nu} p_{3 \lambda}\right)+g_{2 M}^{111} \epsilon_{\nu \lambda \alpha \beta} p_{3}^{\alpha} p_{1}^{\beta}\right]+ \\
& \frac{1}{2}\left(p_{2}-p_{1}\right)_{\nu}\left[g_{3 T}^{111}\left(p_{1} \cdot p_{2} g_{\lambda \mu}-p_{2 \lambda} p_{1 \mu}\right)+g_{3 M}^{111} \epsilon_{\lambda \mu \alpha \beta} p_{1}^{\alpha} p_{2}^{\beta}\right]+ \\
& \frac{1}{8} g_{L}^{111}\left(p_{3}-p_{2}\right)_{\lambda}\left(p_{1}-p_{3}\right)_{\mu}\left(p_{2}-p_{1}\right)_{\nu} \text {. }
\end{aligned}
$$

After a little work the width of the process is calculated to be

$$
\begin{aligned}
\Gamma=\frac{\Delta^{3}}{192 \pi m_{1}^{3}} & \left\{\frac{1}{2 m_{1}^{2}}\left[\left(\Delta^{2}+6 m_{2}^{2} m_{3}^{2}\right)\left|g_{1 T}^{111}\right|^{2}+\Delta^{2}\left|g_{1 M}^{111}\right|^{2}\right]+\frac{\Delta^{4}}{16 m_{1}^{2} m_{2}^{2} m_{3}^{2}}\left|g_{L}^{111}\right|^{2}+\right. \\
& \frac{1}{2 m_{2}^{2}}\left[\left(\Delta^{2}+6 m_{1}^{2} m_{3}^{2}\right)\left|g_{2 T}^{111}\right|^{2}+\Delta^{2}\left|g_{2 M}^{111}\right|^{2}\right]+ \\
& \frac{1}{2 m_{3}^{2}}\left[\left(\Delta^{2}+6 m_{1}^{2} m_{2}^{2}\right)\left|g_{3 T}^{111}\right|^{2}+\Delta^{2}\left|g_{3 M}^{111}\right|^{2}\right]+ \\
& 2 \operatorname{Re}\left(m_{1}^{2} g_{2 T}^{111} g_{3 T}^{111 *}+m_{2}^{2} g_{1 T}^{111} g_{3 T}^{111 *}+m_{3}^{2} g_{1 T}^{111} g_{2 T}^{111 *}\right)+\frac{\Delta^{2}}{2} \operatorname{Re}\left[\left(\frac{g_{1 T}^{111}}{m_{1}^{2}}+\frac{g_{2 T}^{111}}{m_{2}^{2}}+\frac{g_{3 T}^{111}}{m_{3}^{2}}\right) g_{L}^{111 *}\right\}
\end{aligned}
$$

For the particular case that one of the final particles is a photon, say 2, gauge invariance on that leg, $p_{2}^{\mu} \mathcal{M}_{\lambda \mu \nu}=0$, results in three conditions,

$$
g_{L}^{111} \rightarrow 0, \quad g_{2 T}^{111} \rightarrow 0, \quad g_{2 M}^{111} \rightarrow 0,
$$

reducing number of amplitudes for $1 \rightarrow \gamma 1$ to four (which we check presently via the helicity formalism); the corresponding decay width reads

$$
\Gamma^{111} \rightarrow \frac{\Delta^{5}}{384 \pi m_{1}^{3}}\left[\frac{1}{m_{1}^{2}}\left(\left|g_{1 T}^{111}\right|^{2}+\left|g_{1 M}^{111}\right|^{2}\right)+\frac{1}{m_{3}^{2}}\left(\left|g_{3 T}^{111}\right|^{2}+\left|g_{3 M}^{111}\right|^{2}\right)\right], \quad \Delta \rightarrow m_{1}^{2}-m_{3}^{2} .
$$

When both final state particles are photons so that gauge invariance applies to leg 3 as well, only $g_{1 T}^{111}$ and $g_{1 M}^{111}$ survive and one remains with

$$
\Gamma^{111} \rightarrow \frac{m_{1}^{5}}{384 \pi}\left(\left|g_{1 T}^{111}\right|^{2}+\left|g_{1 M}^{111}\right|^{2}\right) .
$$

Examples of such decay processes are $\psi \rightarrow K^{*} \bar{K}^{*}, \psi \rightarrow \gamma \gamma$.

$1 \rightarrow 1 / 2+3 / 2$

We get this from $3 / 2 \rightarrow 1 / 2+1$ by substitution and shall therefore not repeat the expression apart from reminding the reader about the initial spin factor in the partial decay width.

Instances of such decays are rare, but $\psi \rightarrow \bar{\Xi} \Xi^{*}$ is a case in point.

$\underline{1 \rightarrow 3 / 2+3 / 2}$

Substitution allows us to deduce this from $3 / 2 \rightarrow 3 / 2+1$.

Two examples are $\psi \rightarrow \Delta \bar{\Delta}, \Xi^{*} \bar{\Xi}^{*}$.

\section{B. Helicity amplitudes}

$\underline{0 \rightarrow 0+0}$

No analysis is required here. 


$$
M_{0,0}=g^{000} .
$$

$\underline{0 \rightarrow 0+1}$

This case is equally simple:

$$
M_{0,0}=\frac{\Delta}{2 m_{3}} g^{001}
$$

$0 \rightarrow 1 / 2+1 / 2$

When contracting the effective Lagrangian (a crossed version of $1 / 2 \rightarrow 1 / 2+0$ ) against the spinors,

$$
\mathcal{L}=\bar{u}^{\left(\lambda_{2}\right)}\left(p_{2}\right) \mathcal{M} v^{\left(\lambda_{3}\right)}\left(p_{3}\right)
$$

it is worth pointing out that, here and below, one runs across kinematic factors which can be simplified as follows:

$$
\sqrt{\left(E_{3}-m_{3}\right)\left(E_{2} \pm m_{2}\right)}+\sqrt{\left(E_{3}+m_{3}\right)\left(E_{2} \mp m_{2}\right)}=\sqrt{2\left(p_{2} \cdot p_{3} \mp m_{2} m_{3}\right)} .
$$

In this way, for this case, one arrives at the helicity amplitudes,

$$
\begin{aligned}
& \frac{1}{2}\left(M_{1 / 2,1 / 2}+M_{-1 / 2,-1 / 2}\right)=-\sqrt{2 p_{2} \cdot p_{3}-2 m_{2} m_{3}} f^{0 \frac{1}{2} \frac{1}{2}}, \\
& \frac{1}{2}\left(M_{1 / 2,1 / 2}-M_{-1 / 2,-1 / 2}\right)=-i \sqrt{2 p_{2} \cdot p_{3}+2 m_{2} m_{3}} g^{0 \frac{1}{2} \frac{1}{2}} .
\end{aligned}
$$

$\underline{0 \rightarrow 1+1}$

$$
M_{0,0}=m_{2} m_{3} g_{T}^{011}+\frac{\Delta^{2}}{4 m_{2} m_{3}} g_{L}^{011} .
$$

The parity symmetric combinations under final helicity reversal are

$$
\frac{1}{2}\left(M_{1,1}+M_{-1,-1}\right)=p_{2} \cdot p_{3} g_{T}^{011}, \quad M_{1,1}-M_{-1,-1}=i \Delta g_{M}^{011}
$$

$0 \rightarrow 1 / 2+3 / 2$

The contraction here brings in the spin $3 / 2$ antiparticle wavefunctions which are obtained from the direct product topmost state, $v_{\nu}^{(3 / 2)}\left(p_{3}\right) \equiv \varepsilon_{\nu}^{(1) *}\left(p_{3}\right) v^{(1 / 2)}\left(p_{3}\right)$ by spin lowering. As with the decay $0 \rightarrow 1 / 2+1 / 2$, we are restricted to helicity values $\lambda_{3}=\lambda_{2}= \pm 1 / 2$ because of the initial spin. We thereby obtain

$$
\begin{aligned}
& \frac{1}{2}\left(M_{1 / 2,1 / 2}+M_{-1 / 2,-1 / 2}\right)=-\sqrt{\frac{1}{3}\left(p_{2} \cdot p_{3}-m_{2} m_{3}\right)} \frac{\Delta}{m_{3}} f^{0 \frac{1}{2} \frac{3}{2}}, \\
& \frac{1}{2}\left(M_{1 / 2,1 / 2}-M_{-1 / 2,-1 / 2}\right)=-i \sqrt{\frac{1}{3}\left(p_{2} \cdot p_{3}+m_{2} m_{3}\right)} \frac{\Delta}{m_{3}} g^{0 \frac{13}{2}} .
\end{aligned}
$$


Observe how this represents a crossed version of $3 / 2 \rightarrow 1 / 2+0$.

$0 \rightarrow 3 / 2+3 / 2$

There occur four helicity amplitudes now, because $\lambda_{3}=\lambda_{2}$ can run through all values. The results read,

$$
\begin{gathered}
\frac{1}{2}\left(M_{3 / 2,3 / 2}+M_{-3 / 2,-3 / 2}\right)=-\sqrt{2\left(p_{2} \cdot p_{3}-m_{2} m_{3}\right)} p_{2} \cdot p_{3} f_{T}^{0 \frac{3}{2} \frac{3}{2}}, \\
\frac{1}{2}\left(M_{3 / 2,3 / 2}-M_{-3 / 2,-3 / 2)}=-i \sqrt{2\left(p_{2} \cdot p_{3}+m_{2} m_{3}\right)} p_{2} \cdot p_{3} g_{T}^{0 \frac{3}{2} \frac{3}{2}},\right. \\
\frac{1}{2}\left(M_{1 / 2,1 / 2}+M_{-1 / 2,-1 / 2}\right)=-\sqrt{\frac{2}{9}\left(p_{2} \cdot p_{3}-m_{2} m_{3}\right)}\left[\left(p_{2} \cdot p_{3}+2 m_{2} m_{3}\right) f_{T}^{0 \frac{3}{2} \frac{3}{2}}+\frac{\Delta^{2}}{2 m_{2} m_{3}} f_{L}^{0 \frac{3}{2} \frac{3}{2}}\right], \\
\frac{1}{2}\left(M_{1 / 2,1 / 2}-M_{-1 / 2,-1 / 2}\right)=i \sqrt{\frac{2}{3}\left(p_{2} \cdot p_{3}+m_{2} m_{3}\right)}\left[\left(p_{2} \cdot p_{3}-2 m_{2} m_{3}\right) g_{T}^{0 \frac{3}{2} \frac{3}{2}}-\frac{\Delta^{2}}{2 m_{2} m_{3}} g_{L}^{0 \frac{3}{2} \frac{3}{2}}\right] .
\end{gathered}
$$

$\underline{1 \rightarrow 0+0}$

This is a crossed version of an earlier case.

$$
M_{0,0}=\frac{\Delta}{2 m_{1}} g^{100}
$$

$\underline{1 \rightarrow 0+1}$

$$
\begin{gathered}
M_{0,0}=-m_{1} m_{3} g_{T}^{101}-\frac{\Delta^{2}}{4 m_{1} m_{3}} g_{L}^{101} \\
\frac{1}{2}\left(M_{0,1}+M_{0,-1}\right)=-p_{1} \cdot p_{3} g_{T}^{101}, \quad \frac{1}{2}\left(M_{0,1}-M_{0,-1}\right)=i \frac{\Delta}{2} g_{M}^{101} .
\end{gathered}
$$

$1 \rightarrow 1 / 2+1 / 2$

In terms of the covariant electric and magnetic decomposition,

$$
\begin{gathered}
\frac{1}{2}\left(M_{1 / 2,1 / 2}+M_{-1 / 2,-1 / 2}\right)=-\sqrt{2\left(p_{2} \cdot p_{3}-m_{2} m_{3}\right)} \frac{\Delta}{2 m_{1}} f_{E}^{1 \frac{1}{2} \frac{1}{2}}, \\
\frac{1}{2}\left(M_{1 / 2,1 / 2}-M_{-1 / 2,-1 / 2}\right)=-i \sqrt{2\left(p_{2} \cdot p_{3}+m_{2} m_{3}\right)} \frac{\Delta}{2 m_{1}} g_{E}^{1 \frac{1}{2} \frac{1}{2}}, \\
\frac{1}{2}\left(M_{1 / 2,-1 / 2}+M_{-1 / 2,1 / 2}\right)=\sqrt{2\left(p_{2} \cdot p_{3}-m_{2} m_{3}\right)} \frac{\Delta}{2} f_{M}^{1 \frac{1}{2} \frac{1}{2}}, \\
\frac{1}{2}\left(M_{1 / 2,1 / 2}-M_{-1 / 2,-1 / 2}\right)=i \sqrt{2\left(p_{2} \cdot p_{3}+m_{2} m_{3}\right)} \frac{\Delta}{2} g_{M}^{1 \frac{1}{2} \frac{1}{2}} .
\end{gathered}
$$


$\underline{1 \rightarrow 1+1}$

A total of seven amplitudes out of a possible nine survive because the amplitudes $M_{1,-1}, M_{-1,1}$ are excluded by the initial spin magnitude. This agrees of course with other counting methods.

$$
M_{0,0}=-\frac{\Delta}{2} m_{1} m_{2} m_{3}\left(\frac{g_{1 T}^{111}}{m_{1}^{2}}+\frac{g_{2 T}^{111}}{m_{2}^{2}}+\frac{g_{3 T}^{111}}{m_{3}^{2}}+\frac{\Delta^{2}}{4 m_{1}^{2} m_{2}^{2} m_{3}^{3}} g_{L}^{111}\right) .
$$

Well-defined parity combinations are

$$
\begin{gathered}
M_{1,1}+M_{-1,-1}=\frac{\Delta}{m_{1}} p_{2} \cdot p_{3} g_{1 T}^{111}, \quad M_{1,1}-M_{-1,-1}=i \frac{\Delta^{2}}{2 m_{1}} g_{1 M}^{111}, \\
M_{0,1}+M_{0,-1}=i \frac{\Delta^{2}}{2 m_{2}} g_{2 M}^{111}, \quad M_{0,1}-M_{0,-1}=\frac{\Delta}{m_{2}} p_{2} \cdot p_{3} g_{2 T}^{111}, \\
M_{1,0}+M_{-1,0}=i \frac{\Delta^{2}}{2 m_{3}} g_{3 M}^{111}, \quad M_{1,0}-M_{-1,0}=-\frac{\Delta}{m_{3}} p_{2} \cdot p_{3} g_{3 T}^{111} .
\end{gathered}
$$

We can see directly from above that if particle 2 is a photon, the couplings $g_{L}^{111}, g_{2 T}^{111}, g_{2 M}^{111}$, must disappear, like we established before (and $g_{3 T}^{111}=g_{3 M}^{111} \rightarrow 0$ if the third leg is electromagnetic too).

$\underline{1 \rightarrow 1 / 2+3 / 2}$

$$
\begin{aligned}
& \frac{1}{2}\left(M_{1 / 2,3 / 2}+M_{-1 / 2,-3 / 2}\right)=\sqrt{2\left(p_{2} \cdot p_{3}-m_{2} m_{3}\right)} p_{1} \cdot p_{3} f_{T}^{1 \frac{1}{2} \frac{3}{2}}, \\
& \frac{1}{2}\left(M_{1 / 2,1 / 2}+M_{-1 / 2,-1 / 2}\right)=\sqrt{\frac{1}{3}\left(p_{2} \cdot p_{3}-m_{2} m_{3}\right)}\left(2 m_{1} m_{3} f_{T}^{1 \frac{1}{2} \frac{3}{2}}+\frac{\Delta^{2}}{2 m_{1} m_{3}} f_{L}^{1 \frac{1}{2} \frac{3}{2}}\right), \\
& \frac{1}{2}\left(M_{1 / 2,-1 / 2}+M_{-1 / 2,1 / 2}\right)=\sqrt{\frac{1}{3}\left(p_{2} \cdot p_{3}-m_{2} m_{3}\right)}\left(p_{1} \cdot p_{3} f_{T}^{1 \frac{1}{2} \frac{3}{2}}+\frac{\Delta^{2}}{2 m_{3}} f_{M}^{1 \frac{1}{2} \frac{3}{2}}\right), \\
& \frac{1}{2}\left(M_{1 / 2,3 / 2}-M_{-1 / 2,-3 / 2}\right)=i \sqrt{2\left(p_{2} \cdot p_{3}+m_{2} m_{3}\right)} p_{1} \cdot p_{3} g_{T}^{1 \frac{1}{2} \frac{3}{2}} \\
& \frac{1}{2}\left(M_{1 / 2,1 / 2}-M_{-1 / 2,-1 / 2}\right)=i \sqrt{\frac{1}{3}\left(p_{2} \cdot p_{3}+m_{2} m_{3}\right)}\left(2 m_{1} m_{3} g_{T}^{1 \frac{1}{2} \frac{3}{2}}+\frac{\Delta^{2}}{2 m_{1} m_{3}} g_{L}^{1 \frac{1}{2} \frac{3}{2}}\right), \\
& \frac{1}{2}\left(M_{1 / 2,-1 / 2}-M_{-1 / 2,1 / 2}\right)=i \sqrt{\frac{1}{3}\left(p_{2} \cdot p_{3}+m_{2} m_{3}\right)}\left(p_{1} \cdot p_{3} g_{T}^{1 \frac{1}{2} \frac{3}{2}}+\frac{\Delta^{2}}{2 m_{3}} g_{M}^{1 \frac{1}{2} \frac{3}{2}}\right) .
\end{aligned}
$$

$\underline{1 \rightarrow 3 / 2+3 / 2}$ 


$$
\begin{aligned}
& \frac{1}{2}\left(M_{3 / 2,3 / 2}+M_{-3 / 2,-3 / 2}\right)=-\sqrt{2\left(p_{2} \cdot p_{3}-m_{2} m_{3}\right)} \frac{\Delta}{2 m_{1}} p_{2} \cdot p_{3} f_{1 T}^{1 \frac{3}{2} \frac{3}{2}}, \\
& \frac{1}{2}\left(M_{3 / 2,1 / 2}+M_{-3 / 2,-1 / 2}\right)=-\sqrt{\frac{1}{3}\left(p_{2} \cdot p_{3}-m_{2} m_{3}\right)} \Delta\left(\frac{p_{1} \cdot p_{2}}{m_{3}} f_{3 T}^{1 \frac{3}{2} \frac{3}{2}}-f_{M}^{1 \frac{3}{2} \frac{3}{2}}\right), \\
& \frac{1}{2}\left(M_{1 / 2,3 / 2}+M_{-1 / 2,-3 / 2}\right)=-\sqrt{\frac{1}{3}\left(p_{2} \cdot p_{3}-m_{2} m_{3}\right)} \Delta\left(\frac{p_{1} \cdot p_{3}}{m_{2}} f_{2 T}^{1 \frac{3}{2} \frac{3}{2}}-f_{M}^{1 \frac{3}{2} \frac{3}{2}}\right), \\
& \frac{1}{2}\left(M_{1 / 2,-1 / 2}+M_{-1 / 2,1 / 2}\right)=-\sqrt{\frac{1}{9}\left(p_{2} \cdot p_{3}-m_{2} m_{3}\right)} \Delta\left(\frac{p_{1} \cdot p_{3}}{m_{3}} f_{2 T}^{1 \frac{3}{2} \frac{3}{2}}-\frac{p_{1} \cdot p_{2}}{m_{3}} f_{3 T}^{1 \frac{3}{2} \frac{3}{2}}+f_{M}^{1 \frac{3}{2} \frac{3}{2}}\right), \\
& \frac{1}{2}\left(M_{1 / 2,1 / 2}+M_{1 / 2,1 / 2}\right)=-\sqrt{\frac{1}{9}\left(p_{2} \cdot p_{3}-m_{2} m_{3}\right)} m_{1} m_{2} m_{3} \Delta \\
& {\left[\left(1+\frac{p_{2} \cdot 3}{2 m_{2} m_{3}}\right) \frac{f_{1 T}^{1 \frac{3}{2} \frac{3}{2}}}{m_{1}^{2}}+\frac{f_{2 T}^{1 \frac{3}{2} \frac{3}{2}}}{m_{2}^{2}}+\frac{f_{3 T}^{1 \frac{3}{2} \frac{3}{2}}}{m_{3}^{2}}-\frac{\Delta^{2}}{4 m_{1}^{2} m_{2}^{2} m_{3}^{2}} f_{L}^{1 \frac{3}{2} \frac{3}{2}}\right]} \\
& \frac{1}{2}\left(M_{3 / 2,3 / 2}-M_{-3 / 2,-3 / 2}\right)=-i \sqrt{2\left(p_{2} \cdot p_{3}+m_{2} m_{3}\right)} \frac{\Delta}{2 m_{1}} p_{2} \cdot p_{3} g_{1 T}^{1 \frac{3}{2} \frac{3}{2}} \\
& \frac{1}{2}\left(M_{3 / 2,1 / 2}-M_{-3 / 2,-1 / 2}\right)=\sqrt{\frac{1}{3}\left(p_{2} \cdot p_{3}+m_{2} m_{3}\right)} \Delta\left(\frac{p_{1} \cdot p_{2}}{m_{3}} g_{3 T}^{1 \frac{3}{2} \frac{3}{2}}-g_{M}^{1 \frac{3}{2} \frac{3}{2}}\right), \\
& \frac{1}{2}\left(M_{1 / 2,3 / 2}-M_{-1 / 2,-3 / 2}\right)=-i \sqrt{\frac{1}{3}\left(p_{2} \cdot p_{3}+m_{2} m_{3}\right)} \Delta\left(\frac{p_{1} \cdot p_{3}}{m_{2}} g_{2 T}^{1 \frac{3}{2} \frac{3}{2}}+g_{M}^{1 \frac{3}{2} \frac{3}{2}}\right), \\
& \frac{1}{2}\left(M_{1 / 2,-1 / 2}-M_{-1 / 2,1 / 2}\right)=-i \sqrt{\frac{1}{3}\left(p_{2} \cdot p_{3}+m_{2} m_{3}\right)} \Delta\left(\frac{p_{1} \cdot p_{3}}{m_{2}} g_{2 T}^{1 \frac{3}{2} \frac{3}{2}}+\frac{p_{1} \cdot p_{2}}{m_{3}} g_{3 T}^{1 \frac{3}{2} \frac{3}{2}}+\frac{2 p_{2} \cdot p_{3}}{m_{2} m_{3}} g_{M}^{1 \frac{3}{2} \frac{3}{2}}\right), \\
& \frac{1}{2}\left(M_{1 / 2,1 / 2}-M_{1 / 2,1 / 2}\right)=-i \sqrt{\frac{1}{9}\left(p_{2} \cdot p_{3}+m_{2} m_{3}\right)} m_{1} m_{2} m_{3} \Delta \\
& {\left[\left(1-\frac{p_{2} \cdot 3}{2 m_{2} m_{3}}\right) \frac{g_{1 T}^{1 \frac{3}{2} \frac{3}{2}}}{m_{1}^{2}}+\frac{g_{2 T}^{1 \frac{3}{2} \frac{3}{2}}}{m_{2}^{2}}+\frac{g_{3 T}^{1 \frac{3}{2} \frac{3}{2}}}{m_{3}^{2}}-\frac{\Delta^{2}}{4 m_{1}^{2} m_{2}^{2} m_{3}^{2}} g_{L}^{1 \frac{3}{2} \frac{3}{2}}\right]}
\end{aligned}
$$

In all the cases above one may readily verify that the decay width expressions determined previously emerge upon summing over helicity states; this is a helpful independent check.

\section{MAGNITUDES}

The Particle Data Tables contain vast quantities of information about decay processes [5], all amenable to theoretical analysis. Examining every single channel (even for a single particle like $\psi$ ), in order to test theoretical predictions, represents a major undertaking. Many publications have appeared which focus on one aspect or another of such decays, such as semileptonic channels or nonleptonic channels involving a double change in quantum 
numbers. In this paper, we have chosen instead to concentrate on amplitude analysis. For the rest, we shall content ourselves with looking at some typical electromagnetic, weak and strong decays in order to make an important point: that it rather easy to derive the correct partial decay width, within a factor of about two ( $\sim$ a Clebsch-Gordan coefficient $)$, just by incorporating the proper phase space, CKM angles and invoking the magnitudes,

$$
g_{\text {strong }} \sim 10, \quad e \simeq 0.3, \quad G_{F} m_{p}^{2} / 2 \sqrt{2}=3.62 \times 10^{-6} .
$$

Our main conclusion is that a stringent test of theoretical predictions demands that experimental fits should be good to within $10 \%$ at least; otherwise there is little point in extolling the virtues of one theoretical model over another.

To substantiate this statement, look at weak processes $0^{-} \rightarrow 0^{-} 0^{-}$and $0^{-} \rightarrow 0^{-} 1^{-}$, which are governed by a single coupling constant, whose magnitude can be directly obtained from the decay width. Because, as defined, $g^{000}$ has dimensions, we shall rescale it to a dimensionless number by dividing it by the mass of the decaying particle - this seems the most natural choice. ( $g^{001}$ is automatically dimensionless and does not need massaging.) For $B$-meson decays, we further divide out the CKM matrix elements, $\left|V_{c b}\right|$ and $\left|V_{u d}\right|$ or $\left|V_{c s}\right|$, which we anticipate to be indirectly present from the current-current picture model; so for example, one should understand that $g^{B D \pi}=g^{000}(B \rightarrow D \pi) / m_{B} V_{c b} V_{u d}$ below, etc. The results for typical $B$-meson decays read (in units of $10^{-6}$ )

$$
\begin{aligned}
& g^{B^{+} D^{0} \pi^{+}}=3.84(0.12) \quad g^{B^{0} D^{-} \pi^{+}}=2.94(0.14) \\
& g^{B^{+} D^{* 0} \pi^{+}}=3.42(0.15) \quad g^{B^{0} D^{*-} \pi^{+}}=2.46(0.15) \\
& g^{B^{+} \psi \pi^{+}}=3.15(0.42) \\
& g^{B^{+} \psi K^{+}}=3.48(0.32), \quad g^{B^{0} \psi K^{0}}=3.06(0.39) \\
& g^{B^{+} D^{0} \rho^{+}}=2.13(0.14) \quad g^{B^{0} D^{-} \rho^{+}}=1.66(0.16),
\end{aligned}
$$

where the entries in the parenthesis are relative errors derived from the uncertainties of the measured branching ratios and the elements of the Cabibbo-Kobayashi-Maskawa matrix. We see that the couplings $g^{B D \pi}$ along with $g^{B \psi \pi}$ and $g^{B \psi K}$ hover around 3, while the couplings $g^{B D \rho}$ are somewhat smaller. These values are comparable to $m_{p}^{2} G_{F} / 2 \sqrt{2}$.

With respect to strong processes, parity is conserved and there are fewer independent couplings as a result. From the data one determines the following typical coupling constants,

$$
\begin{aligned}
& g^{K^{*} K \pi}=11.2 \\
& f^{\Delta N \pi}=10.9 \\
& g^{N^{*} \pi}=9.65, \quad N^{*}=N(1440) .
\end{aligned}
$$

Here, $f^{\frac{3}{2} \frac{1}{2} 0}$ has been rescaled by a factor $m_{\Delta}$ while $g^{\frac{1}{2} \frac{1}{2} 0}$ is already dimensionless. These numbers are typical of strong couplings, as we have asserted above.

Coupling constants for electromagnetic processes of ground state hadrons accord well[ with supermultiplet predictions [6], so it is of interest to look for the effects connected with excitations; in particular the coupling,

\footnotetext{
${ }^{3}$ In this connection we note that relativistic spin-flavour symmetry produces a particular invariant amplitude, which can be re-expressed in terms our previous amplitudes, via (with $m_{3}=0$ ), $\left(m_{1}-m_{2}\right)^{2} \varepsilon^{* 3 \nu} \bar{u}_{2} \epsilon_{\nu \alpha \beta \lambda} p_{1}^{\alpha} p_{2}^{\beta} u_{1}^{\lambda}=\varepsilon^{* \nu} \bar{u}_{2}\left[\left(p_{1} \cdot p_{3} g_{\lambda \nu}-p_{3 \lambda} p_{1 \nu}\right) \gamma_{5}\left(m_{1}^{2}-m_{2}^{2}\right)-m_{1}\left(p_{3}-p_{2}\right)_{\lambda} \epsilon_{\nu \alpha \beta \rho} p_{1}^{\alpha} p_{2}^{\beta} \gamma^{\rho}\right] u_{1}^{\lambda}$,
} 


$$
f_{M}^{N^{*} p \gamma}=0.0988
$$

which has been rescaled by a factor of $m_{N^{*}}^{2}$, is smaller than electromagnetic value magnitude $e$; this is not surprising because it represents a transition between an excited and a ground state - it should therefore be smaller than an electromagnetic transition element between $\ell=0$ hadron states. In fact the larger the excitation number difference (and hence the baryon mass values) the smaller do we anticipate the coupling to be, so all is crudely in order here.

In summary, we suggest that all decay analyses, including nonleptonic weak decays, be converted into the covariant decompositions prescribed in the text, for reasons of economy and elegance, and without assuming factorization. As well, we believe that theoretical models (that can be made to accord with data all too readily) will be better differentiated by more accurate experimental results; at the moment there is little to choose between them.

\section{APPENDIX A: NOTATION}

We use a $(+,-,-,-)$ Minkowskian metric $g$ and the state normalization

$$
2 \pi \delta\left(p^{2}-m^{2}\right)\left\langle p^{\prime} j^{\prime} \lambda^{\prime} \mid p j \lambda\right\rangle=(2 \pi)^{4} \delta^{4}\left(p^{\prime}-p\right) \delta_{j^{\prime} j} \delta_{\lambda \lambda^{\prime}}
$$

or

$$
\left\langle\mathbf{p}^{\prime} j^{\prime} \lambda^{\prime} \mid \mathbf{p} j \lambda\right\rangle=2 p_{0}(2 \pi)^{3} \delta^{3}\left(\mathbf{p}^{\prime}-\mathbf{p}\right) \delta_{j j^{\prime}} \delta_{\lambda^{\prime} \lambda} .
$$

We shall denote the intrinsic parity of a state by $\eta$, as determined by strong interactions. A two-body decay process of the type $1 \rightarrow 2+3$ is viewed with all momenta flowing outwards (to ensure that any symmetry and crossing properties are readily implemented); thus to an incoming decaying particle 1 is ascribed a momentum $-p_{1}$, whereas the outgoing momenta are simply written $p_{2}$ and $p_{3}$. While it may seem clumsy to have the negative of one momentum physical, the dividend is that some results assume an elegant form, making it easy easy to permute vertex legs and obtain results in other channels.

Within the covariant formalism, we associate wavefunction $\phi^{*}(p)$ with an outgoing and $\phi(p)$ with an incoming particle of momentum $p$. For spin 0 particless we just interpret

$\phi \rightarrow 1$; for spin $\frac{1}{2}$ we use the traditional Dirac particle wavefunctions $u^{(\lambda)}(p)$ and $\bar{u}^{(\lambda)}(p)$ interpretation, with normalization $\sum_{\lambda} u^{(\lambda)}(p) \bar{u}^{(\lambda)}(p)=\gamma \cdot p+m$; with spin one we understand $\phi$ to be the standard polarization vector, $\varepsilon_{\mu}^{(\lambda)}(p)$, having $\sum_{\lambda} \varepsilon_{\mu}^{(\lambda) *}(p) \varepsilon_{\nu}^{(\lambda)}(p)=-g_{\mu \nu}+p_{\mu} p_{\nu} / m^{2}$, dropping the last term for photons (when amplitudes obey gauge constraints). With excited baryon states we regard $\phi$ as the Rarita-Schwinger wavefunction; so for spin $3 / 2$ we take $\phi$ to be $u_{\mu}^{(\lambda)}(p)$, with normalization

$$
\sum_{\lambda} u_{\mu}^{(\lambda)}(p) \bar{u}_{\nu}^{(\lambda)}(p)=\left(-g_{\mu \nu}+\frac{p_{\mu} p_{\nu}}{m^{2}}\right)(\gamma \cdot p+m)-\frac{1}{3}\left(\gamma_{\mu}+\frac{p_{\mu}}{m}\right)(\gamma \cdot p-m)\left(\gamma_{\nu}+\frac{p_{\nu}}{m}\right) .
$$

Last, but not least, the spinorial wavefunction for an outgoing antifermion is written as $v^{(\lambda)}(p)$, with normalization $\sum_{\lambda} v^{(\lambda)}(p) \bar{v}^{(\lambda)}(p)=\gamma \cdot p-m$, and an analogous expression for the spin $3 / 2$ case exists. It is formally true that the antifermion wavefunction $v^{(\lambda)}(p)=$ 
$(-1)^{j+\lambda} u^{(\lambda)}(-p)=i C \tilde{\bar{u}}^{(\lambda)}(p)$, can be deduced by appropriate continuation from the fermion wavefunction - a result which is comparable to that applying to polarization vectors, viz. $\varepsilon^{(\lambda)}(-p)=(-1)^{j-\lambda} \varepsilon^{(-\lambda) *}(p)$. This leads to simple substitution rules, as is well-known from field theory, and we shall take advantage of that fact in the text.

When constructing covariant $\mathcal{M}$-functions, to be contracted over external wavefunctions, we must first count how many independent couplings are needed. One may deduce their number by the traditional $l-s$ method or by performing a helicity amplitude analysis (see below). A more crossing-symmetric procedure is to count how many ways an integer angular momentum can be obtained by combining spins $\mathbf{j}_{1}, \mathbf{j}_{2}, \mathbf{j}_{3}$, since this does not commit one to any particular channel. The result of such an analysis is as follows: if one can construct a Euclidean triangle with sides $j_{1}, j_{2}, j_{3}$ out of the spins, the number of independent couplings is $N=j_{1}\left(1-j_{1}\right)+j_{2}\left(1-j_{2}\right)+j_{3}\left(1-j_{3}\right)+2\left(j_{1} j_{2}+j_{2} j_{3}+j_{3} j_{1}\right)+1$; Otherwise reorder the spins in order of decreasing magnitude, $j_{a} \geq j_{b} \geq j_{c}$, whereupon $N=\left(2 j_{b}+1\right)\left(2 j_{c}+1\right)$, with $j_{a} \geq j_{b}+j_{c}$. (Because we are mainly considering weak processes, parity conservation is a secondary issue.) Having settled on the number of vertex amplitudes that are needed, we have to determine appropriate tensors (and matrices for spinors) which they multiply. This is where a certain amount of freedom exists; for the equations of motion obeyed by the external wavefunctions allow us to transmogrify a tensor/matrix form into a combination of other forms. A good example of this freedom is to be found in the interaction between a vector meson and two spin $1 / 2$ baryons. Sandwiched between wavefunctions $\bar{u}\left(p^{\prime}\right)$ and $u(p) \varepsilon^{\mu}\left(p^{\prime}-p\right)$, the vectors arising in the $\mathcal{M}_{\mu}$-function are connected in four ways:

$$
\begin{aligned}
\left(p+p^{\prime}\right)_{\mu} & =\left(m+m^{\prime}\right) \gamma_{\mu}+i \sigma_{\mu \nu}\left(p-p^{\prime}\right)^{\nu} \quad(\text { Gordon relation) } \\
\left(m^{\prime}-m\right) \gamma_{\mu} & =i \sigma_{\mu \nu}\left(p+p^{\prime}\right)^{\nu}, \quad(\rightarrow 0 \quad \text { for equal masses }) \\
\left(p-p^{\prime}\right)^{2} \gamma_{\mu} & =i\left(m+m^{\prime}\right) \sigma_{\mu \nu}\left(p-p^{\prime}\right)^{\nu}+2 \epsilon_{\mu \alpha \beta \rho} p^{\alpha} p^{\prime \beta} \gamma^{\rho} \gamma_{5} \\
\left(p+p^{\prime}\right)^{2} \gamma_{\mu} & =\left(m+m^{\prime}\right)\left(p+p^{\prime}\right)_{\mu}+i\left(m^{\prime}-m\right) \sigma_{\mu \nu}\left(p+p^{\prime}\right)^{\nu}-2 \epsilon_{\mu \alpha \beta \rho} p^{\alpha} p^{\prime \beta} \gamma^{\rho} \gamma_{5}
\end{aligned}
$$

(There are similar relations for axial mesons.) We have "experimented" with various tensors/matrices and derived what we believe is the most elegant irreducible set in the main body of the text. This can be confirmed by deriving the helicity amplitudes in terms of the covariant ones and verifying their simplicity. An added advantage of the covariant formalism is that kinematic threshold factors arise naturally once the contractions over wavefunctions are carried out in any given channel. Gauge invariance conditions on the tensors of course lead us to discard certain couplings - in the helicity formalism it is equivalent to ignoring projections onto certain helicity states (longitudinal for photons, right-handed for neutrinos).

The connection with the helicity state formalism is obtained as follows. In the centre of mass frame of the decaying particle, with the relative 3-momentum of the outgoing particles inclined at angle $\theta$ with respect to the spin quantization axis - which we are always free to choose - the $T$-matrix amplitude is expressed as

$$
\left\langle p_{2} \lambda_{2} ; p_{3}, \lambda_{3}|T|-p_{1}, \lambda_{1}\right\rangle \equiv(2 \pi)^{4} \delta^{4}\left(p_{1}+p_{2}+p_{3}\right) d_{\lambda_{1} \lambda_{2}-\lambda_{3}}^{j_{1}}(\theta) M_{\lambda_{2}, \lambda_{3}},
$$

wherein $M_{\lambda_{2}, \lambda_{3}}$ is the reduced matrix element of the decay amplitude and the rotation matrix $d$ obeys the symmetry properties,

$$
d_{\lambda \lambda^{\prime}}^{j}(\theta)=(-1)^{\lambda^{\prime}-\lambda} d_{\lambda^{\prime} \lambda}^{j}(\theta)=d_{-\lambda^{\prime}-\lambda}^{j}(\theta)=d_{\lambda^{\prime} \lambda}^{j}(-\theta) .
$$


Tables of these $d$ can be found in ref [5]. It is worth remarking that all helicity values of the decay products may not be possible in view of the fact that $\left|\lambda_{2}-\lambda_{3}\right|$ cannot exceed $j_{1}$. In the main body of the paper we obtain the connection between the covariantly-defined couplings and the reduced helicity amplitudes; these should not be excessively complicated if the covariant decompositions are properly chosen and indeed serves as a useful guide on how they should be carried out.

So far as discrete space-time symmetries are concerned, if parity happens to be conserved,

$$
M_{\lambda_{2}, \lambda_{3}}=\eta_{1} \eta_{2} \eta_{3}(-1)^{j_{2}+j_{3}-j_{1}} M_{-\lambda_{2},-\lambda_{3}}
$$

Time reversal invariance gives no useful information for decays, nor does charge conjugation invariance, unless the decay products are uncharged and self-conjugate. However if the final pair (2\&3) of particles are identical, we must ensure that the spin-statistics relation, $M_{\lambda_{2}, \lambda_{3}}=(-1)^{j_{1}-2 j_{2}} M_{\lambda_{3}, \lambda_{2}}$ is satisfied. Finally we stress again that certain helicity values may be absent for massless particles.

Observables are obtainable from the $M_{\lambda, \lambda^{\prime}}$. One of the most important of these is associated with the sum over all spins, $\sum_{\lambda_{2}, \lambda_{3}}\left|M_{\lambda_{2}, \lambda_{3}}\right|^{2}$, since the partial decay width for $1 \rightarrow 2+3$ is found by averaging over the initial spins and including a phase-space factor for the final outgoing pair,

$$
\Gamma=\frac{\Delta}{16 \pi\left(2 j_{1}+1\right) m_{1}^{3}} \sum_{\lambda_{2}, \lambda_{3}}\left|M_{\lambda_{2}, \lambda_{3}}\right|^{2} .
$$

Here the symmetrical Kallen triangle function,

$$
\Delta=\sqrt{m_{1}^{4}+m_{2}^{4}+m_{3}^{4}-2 m_{1}^{2} m_{2}^{2}-2 m_{2}^{2} m_{3}^{2}-2 m_{3}^{2} m_{1}^{2}}=2 m_{1} q=2 \sqrt{\left(p_{2} \cdot p_{3}\right)^{2}-m_{2}^{2} m_{3}^{2}},
$$

is proportional to the relative momentum $q$ between $2 \& 3$ in the centre of mass frame of 1 (or in other crossed channels, by permutation of labels) 1 . Other observables are connected with polarizations and come through density matrices of the type $\rho_{\lambda^{\prime} \lambda^{\prime \prime}} \propto \sum_{\lambda} M_{\lambda, \lambda^{\prime}} M_{\lambda, \lambda^{\prime \prime}}^{*}$ which often require analysis of the subsequent interactions or decays of one of the outgoing particles. An alternative way of extracting individual amplitudes is to examine the full angular distributions of the decay products or, with less precision, various asymmetry parameters.

\section{APPENDIX: ACKNOWLEDGMENTS}

This research was supported by a University of Tasmania Special Grant.

\footnotetext{
${ }^{4}$ In this connection, recall that powers of $\Delta^{2 \ell+1}$ which arise from spin summations are connected with the relative orbital momentum $\ell$ of the final particles in the centre of mass frame of the decaying particle.
} 


\section{REFERENCES}

* Email: bob.delbourgo@utas.edu.au

$\dagger$ Email: d.liu@utas.edu.au

[1] N. Isgur and M.B. Wise, in Heavy Flavours, ed. by A.J. Buras and M. Lindner (World Scientific, Singapore, 1992); M. Neubert, Phys. Rep. 245, 259 (1994); M.G. Olsson, preprint MADPH-97-104 or hep-ph/9707283, Conference on B-physics and CP violation, Honolulu (March 1997).

[2] M. Wirbel, Prog. Nucl. Part. Phys. 21, 333 (1988); J.G. Korner, in Baryons '95, ed. by P.D. Barnes et al. (World Scientific, Singapore, 1996); J.G. Korner, D. Pirjol and M. Kramer, Prog. Part. Nucl. Phys. 33, 757 (1990); J.D. Richman and P.R. Burchat, Rev. Mod. Phys. 67, 893 (1996); T.E. Browder, K. Honschied and D. Pedrini, Ann. Rev. Nucl. \& Part. Sc. 46, 395 (1996).

[3] B. Stech, preprint hep-ph/9709280, in b20 Symposium: Twenty Beautiful Years of Bottom Physics, Chicago (July, 1997); M. Neubert, preprint hep-ph/9707368, in Euroconference on QCD, Montpellier (July 1997); R.C. Verma, preprint hep-ph/9703223, in XII DAE Symposium on High Energy Physics, Duhawati (January 1997); M.A. Ivanov, J.G. Korner, V.E. Lyuobovitskij and A.G. Rusedsky, preprint hep-ph/9709325 and MZ-TH/97-21 (September 1997);

[4] M. Bauer, B. Stech and M. Wirbel, Z. Phys. C34, 103 (1987); J.G. Korner and M. Kramer, Z. Phys. C55, 659 (1992); B. Bajc, S. Fajfer and R.J. Oakes, Phys. Rev. D53, 4957 (1996);

[5] R.M. Barnett et al., Review of Particle Properties, Phys. Rev. D54, 1 (1996).

[6] R. Delbourgo and D. Liu, Phys. Rev. D53, 6576 (1996). 